\title{
The Mercury Laser Altimeter Instrument for the MESSENGER Mission
}

John F. Cavanaugh ${ }^{1}$, James C. Smith ${ }^{1}$, Xiaoli Sun ${ }^{1}$, Arlin E. Bartels ${ }^{1}$, Luis RamosIzquierdo $^{2}$, Danny J. Krebs ${ }^{1}$, Anne Marie Novo-Gradac ${ }^{1}$, Jan F. McGarry ${ }^{1}$, Raymond Trunzo $^{4}$, Jamie L. Britt ${ }^{1}$, Jerry Karsh ${ }^{1}$, Richard B. Katz ${ }^{1}$, Alan Lukemire ${ }^{6}$, Richard Szymkiewicz $^{5}$, Daniel L. Berry ${ }^{1}$, Joseph P. Swinski ${ }^{1}$, Gregory A. Neumann ${ }^{1}$, Maria T. Zuber $^{2}$, David E. Smith ${ }^{1}$

Author affiliations:

1. NASA Goddard Space Flight Center

2. Massachusetts Institute of Technology

3. LRI Optical Design

4. Swales Aerospace Corp.

5. Orbital Sciences Corp.,

6. Spacepower, Inc.

\begin{abstract}
The Mercury Laser Altimeter (MLA) is one of the payload science instruments on the MErcury Surface, Space ENvironment, GEochemistry, and Ranging (MESSENGER) mission, which launched on 3 August 2004. The altimeter will measure the round trip time-of-flight of transmitted laser pulses reflected from the surface of the planet that, in combination with the spacecraft orbit position and pointing data, gives a high-precision measurement of surface topography referenced to Mercury's center of mass. The altimeter measurements will be used to determine the planet's forced librations by tracking the motion of large-scale topographic features as a function of time. MLA's laser pulse energy monitor and the echo pulse energy estimate will provide an active measurement of the surface reflectivity at $1064 \mathrm{~nm}$. This paper describes the instrument design, prelaunch testing, calibration, and results of post-launch testing.
\end{abstract}

\section{Introduction}

\subsection{Mission Overview}

The Mercury Laser Altimeter (MLA) is one of seven scientific instruments on the MErcury Surface, Space ENvironment, Geochemistry, and Ranging (MESSENGER) spacecraft. MESSENGER was launched on August 3; 2004, and will enter Mercury orbit in 2011 to perform scientific measurements for a period of one Earth year, equivalent to four Mercury years. MESSENGER will be in a highly elliptical and near-polar orbit around Mercury with a periapsis altitude of 200 to $400 \mathrm{~km}$, an apoapsis altitude of $\sim 15,000 \mathrm{~km}$, and a period of 12 hours. Figure 1 shows the MESSENGER orbits and MLA measurement geometry. The spacecraft altitude will come within the MLA ranging capability near periapsis for 15 to 45 minutes during each orbit, depending on the time of the year. The orbit axis is tilted $25^{\circ}$ from the polar axis to increase the measurement coverage for lowlatitude regions. The intense heat from the Sun will require the spacecraft to have its sunshade facing the Sun at all times, which during noon-midnight orbits will confine the payload deck to point within $10^{\circ}$ about the normal to Mercury's orbital plane with MLA ranging at a slant angle as high as 50 degrees (Solomon et al., 2001). 


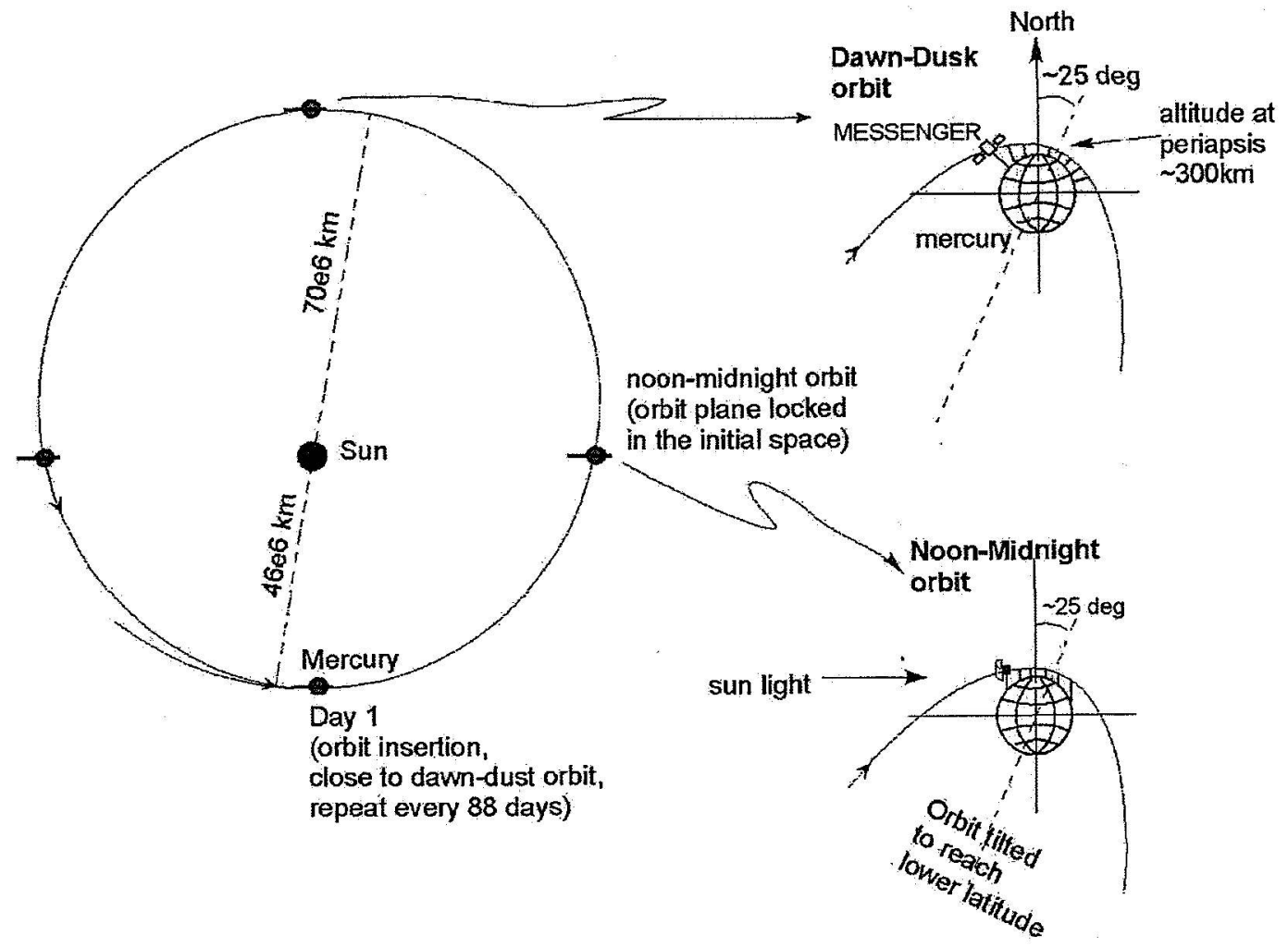

Figure 1 MESSENGER Orbit

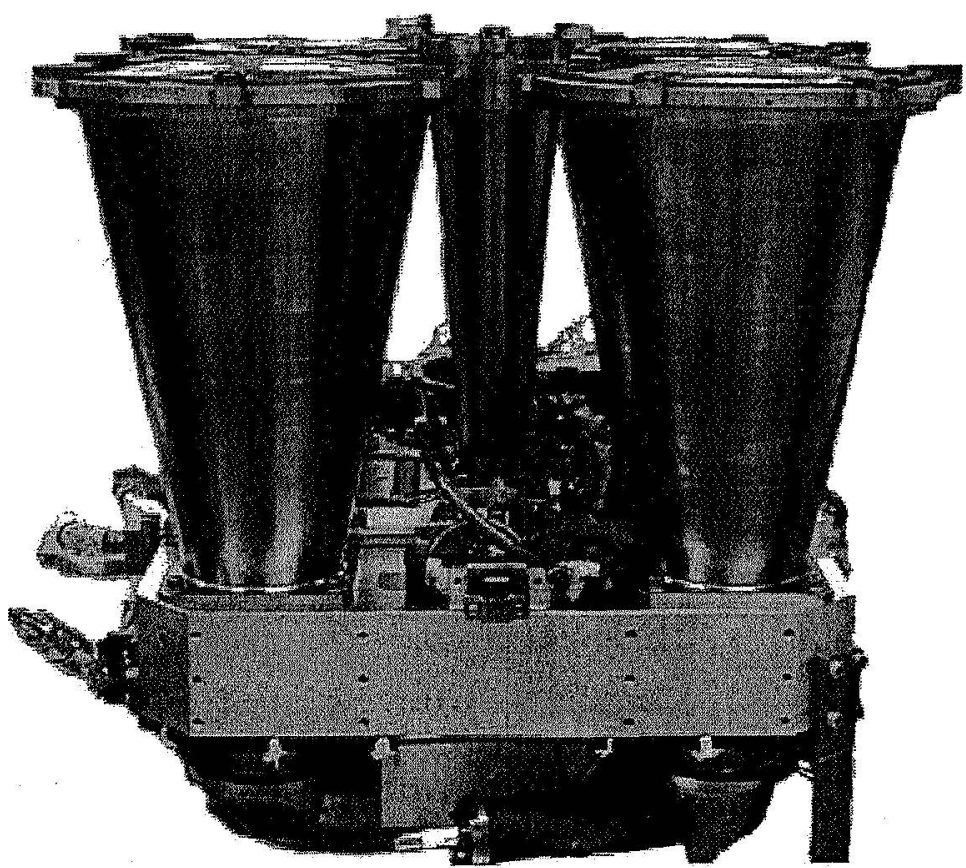

Figure 2 MLA Instrument 


\subsection{MLA Instrument Overview}

MLA is a time-of-flight laser rangefinder that uses direct pulse detection and pulse edge timing to determine range to the surface. Its laser transmitter emits 5-ns pulses at an $8-\mathrm{Hz}$ rate with $20 \mathrm{~mJ}$ of energy at a wavelength of $1064 \mathrm{~nm}$. Return echoes are collected by an array of four refractive telescopes and detected using a single silicon avalanche photodiode and three matched low-pass electronic filters. Pulse timing is measured using a unique combination of crystal-oscillator-based counters and high-resolution time-offlight application-specific integrated circuits (ASICs). The MLA instrument is shown in Figure 2.

\section{Science and Measurement Objectives}

\subsection{MLA Science Objectives}

The primary science measurement objectives for MLA are to provide a high-precision topographic map of the north polar region, measure the long-wavelength topographic features of the mid-to-low latitude region, determine topographic profiles across major geologic features in the northern hemisphere, and detect and quantify the planets forced librations by tracking the motion of large-scale topographic features as a function of time. An additional goal of the MLA instrument is to measure the surface reflectivity of Mercury at the MLA operating wavelength of $1064 \mathrm{~nm}$ (Sun et al., 2004).

\subsection{MLA Instrument Objectives}

MLA will measure the topography of the Mercury northern hemisphere via laser pulse time-of-flight data and spacecraft orbit position data in an approach similar to the Mars Orbiter Laser Altimeter (MOLA) (Abshire, Sun, and Afzal, 2000) . MLA is designed to perform range timing measurements up to $1800 \mathrm{~km}$ from the planet's surface. Since the single pulse signal link margin is close to $0 \mathrm{~dB}$ for altitudes above $800 \mathrm{~km}$, the MLA data acquisition scheme allows collection and downlink of up to fifteen returns per shot in order to allow the use of correlation techniques to process the range signals on the ground. Measurement of both the outgoing pulse energy and received pulse shape enable the surface reflectivity measurement. The MLA functional requirements are enumerated in Table 1; allocations and constraints are listed in Table 2.

\begin{tabular}{l|}
\hline Time stamp laser pulses to better than $\pm 1 \mathrm{~ms}$ with respect to the ephemeris \\
\hline Support determination of pointing stability to $\pm 50 \mathrm{rad}$ peak-to-peak \\
\hline Measure laser pulse time of flight from 1 to $8 \mathrm{~ms}$ with return pulse widths from 6 to $1000 \mathrm{~ns}$ \\
\hline Total ranging error $<1 \mathrm{~m}$ with probability of detection $\mathrm{P}_{\mathrm{d}}>95 \%$ at $200 \mathrm{~km}$ altitude nadir pointing \\
\hline Laser pulse repetition rate of $8 \mathrm{~Hz}$ \\
\hline Produce laser footprint $=10 \mathrm{~m}$ diameter at $200 \mathrm{~km}$ altitude, nadir pointing \\
\hline $\mathrm{P}_{\mathrm{d}}>10 \%$ at $800 \mathrm{~km}$ slant range and $60^{\circ}$ angle \\
\hline Maintain long term ranging bias error to $\leq 0.50 \mathrm{~m}$ over the mission lifetime. \\
\hline Measure the reflectivity of the ranging targets.
\end{tabular}

Table 1 MLA Functional Requirements 


\begin{tabular}{|c|c|}
\hline Parameter & Requirement \\
\hline Instrument design life & 5 -year cruise with power off followed by 1 year of operation \\
\hline Orbit definition & $\begin{array}{l}\text { Periapsis altitude }=200 \mathrm{~km} \text {, apoapsis altitude }=15,193 \mathrm{~km} \text {, } \\
\text { inclination }=80^{\circ} \text {, latitude of periapsis } \leq 60^{\circ} \text { Period }=12 \text { hours }\end{array}$ \\
\hline Flight environment & $\begin{array}{l}\text { Mercury albedo and infrared flux onto the instrument for a } 60 \\
\text { minute pass centered about periapsis. Cold space viewing for } \\
\text { the remaining } 11 \text { hours of the orbit }\end{array}$ \\
\hline Testing environment & $\begin{array}{l}\text { MESSENGER Component Environmental Specification, } \\
\text { drawing \# 7384-9101 }\end{array}$ \\
\hline Mass & $6.1 \mathrm{~kg}$ (allocated) \\
\hline Power & $\begin{array}{l}25 \mathrm{~W} \text { for } 60 \text { minutes centered on periapsis and } 11 \mathrm{~W} \text { for the } \\
\text { remaining } 11 \text { hours of the orbit. }\end{array}$ \\
\hline Telemetry & 2.0 Mbits average per 12 hour orbit about Mercury \\
\hline Time correlation & $\begin{array}{l}1 \text { pulse per second time tick from the spacecraft along with } \\
\text { announcement with }<50 \mathrm{~ms} \text { accuracy in real time and }<1 \mathrm{~ms} \\
\text { post navigation data processing }\end{array}$ \\
\hline Relative pointing & $\begin{array}{l}\text { Determine laser output pointing direction with respect to the } \\
\text { spacecraft axes over temperature to } \pm 50 \mu \mathrm{rad} \text { peak to peak }\end{array}$ \\
\hline Envelope & $\begin{array}{l}28 \mathrm{~cm} \times 28 \mathrm{~cm} \times 26 \mathrm{~cm} \text { initial allocation; envelope to be } \\
\text { captured in mechanical ICD }\end{array}$ \\
\hline
\end{tabular}

Table 2 MLA Allocations and Constraints

\section{MLA Instrument Design}

\subsection{Background}

MLA builds on the experiences gained from the development of several space-based and airborne laser altimeter systems flown over the past two decades including the Mars Observer Laser Altimeter MOLA-1 (Zuber et al., 1992), the Mars Orbiter Laser Altimeter MOLA-2 (Smith, et al., 1998)), two Shuttle Laser Altimeters SLA-1 and SLA2 (Garvin, et al., 1998), the Geoscience Laser Altimeter System on the Ice Cloud and Elevation Satellite GLAS/ICESAT (Zwally et al., 2002), and the airborne Microchip Laser Altimeter System Microaltimeter (Degnan, 2002). The measurement scheme for MLA is similar to that of MOLA, in which the outgoing pulse starts a counter that is stopped by the detection of a received echo pulse. A significant change implemented in MLA is the ability to record several return pulses, process them on board and send the "most likely" signal returns to the ground for correlation analysis. This technique is similar to and derived from experience with the Microaltimeter (Degnan, 2002).

\subsection{MLA Functional Overview}

Refer to the MLA functional block diagram Figure 3 and the system timing diagram Figure 4. A trigger pulse initiated by the range measurement electronics starts the optical pumping of the MLA laser. This trigger pulse also initiates a 5-MHz 23-bit counter, 16 bits of which are used for coarse range timing. After optically pumping the laser for approximately $150 \mu$ s the laser emits a $6-\mathrm{ns} 20-\mathrm{mJ}$ pulse, which propagates to the planet's surface. A photodiode and a comparator detect the emitted pulse. The leading edge of 
this pulse starts the first of six fine-resolution time-of-flight counters, which is subsequently stopped by the next edge of the $5-\mathrm{MHz}$ clock. These counters provide a relative time within each $200 \mathrm{~ns}$ coarse clock cycle. The coarse counter value is latched at this point, and the two counters provide the leading-edge start timing value for the range measurement. The trailing edge of the start pulse is timed in an identical manner. These first two counts establish the start time of the range timing cycle. The pulse width is computed by subtracting the leading edge measurement from the trailing edge measurement.

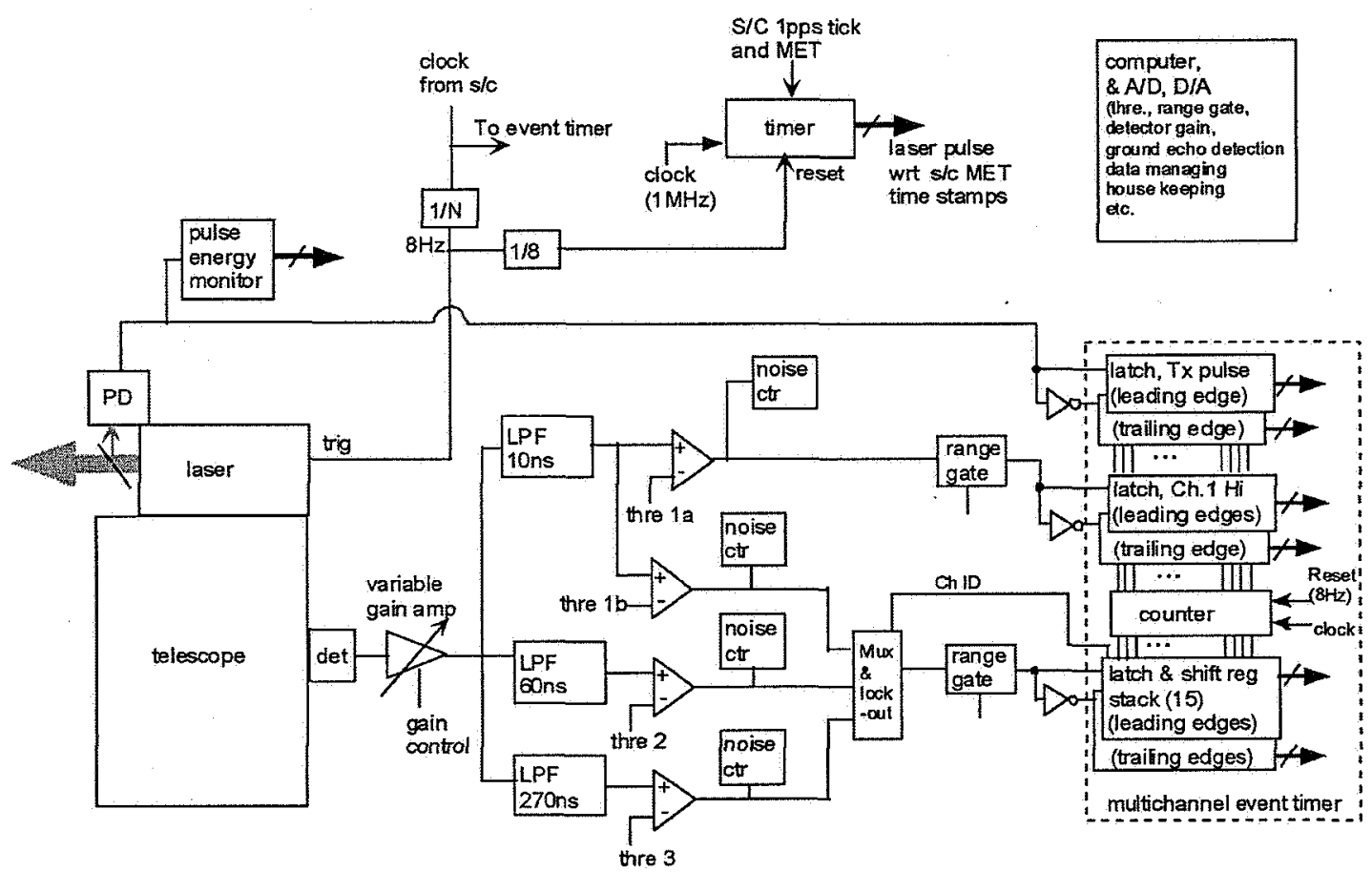

Figure 3 MLA Functional Block Diagram

A portion of the pulse energy reflected off the planet's surface is collected by MLA's four receiver telescopes and focused onto four optical fibers which relay the signal through an optical bandpass filter to a silicon avalanche photodiode (SiAPD) which preserves to the extent of its bandwidth the deformation of the incident pulse caused by interaction with surface features. Slant angle ranging, surface roughness or large-scale features on the surface can cause this temporal pulse spreading. To maximize the probability of detecting spread returns, three matched filters are used after the detector amplifier. The high-bandwidth filter output, with a 10-ns impulse response, is split and fed to two comparators with independently programmable thresholds referred to as channel 1 high and low respectively. The channel 1 high comparator signal is fed to the second set of time-of-flight counters, which record the leading and trailing edge times of this pulse. The channel 1 low comparator and the other filter outputs with $60-n$ s and $270-$ ns impulse response, referred to as channels 2 and 3, respectively, are fed to the third set of counters. For this last set of counters the first leading edge to stop a counter locks out the other channels for approximately $1 \mu \mathrm{s}$ in order to prevent a different channel from 
stopping the trailing edge counter. The MLA Range Measurement Unit (RMU) can collect and store up to fifteen pulse returns within the range gate from this last set of counters.

The nominal operational timebase for MLA event sequencing and range measurement is the MESSENGER spacecraft's oven-controlled crystal oscillator. The 5-MHz signal from either of the spacecraft's redundant oscillators can be used. The laser trigger operating at $8 \mathrm{~Hz}$ is also synchronized to the spacecraft 1-pulse-per-second timing reference. A third backup 5-MHz crystal oscillator on the MLA RMU board can also be used.

To maximize the probability of return signal detection in MESSENGER's dynamic operating environment MLA uses a real-time embedded algorithm to monitor background noise and adjust comparator threshold levels to minimize false signals. The MESSENGER spacecraft also provides MLA with once-per-second updates of estimated line-of-sight range to Mercury based on spacecraft attitude and orbit data. This information is used to set the range gate time delays and gain levels for the detector amplifier.

For altitudes greater than $800 \mathrm{~km}$ on the inbound and outbound orbit trajectories the MLA signal-to-noise ratio becomes sufficiently weak that the "high" threshold channel is no longer detecting the return. In this situation the software collects and downlinks the multiple return pulses from the "low" channel timers.

The signal tracking algorithm and the MLA command and data handling software are implemented on a radiation-hardened $80 \mathrm{C} 196$ processor that, with a control and data communications field-programmable gate array (FPGA) and memory devices, comprise the central-processing unit (CPU) electronics board. The RMU board is housed with this board in a box underneath the main instrument housing (Figure 2). The detector, amplifiers, and comparators are mounted on a separate printed wiring board and mechanically mated to the aft optics, which filter and focus the signal from the receiver fiber optics.

Housekeeping signal conditioning and data conversion functions are performed on another circuit board referred to as the Analog Electronics Module (AEM). Laser control and drive functions are implemented on two assemblies beneath the laser bench referred to as the Laser Electronics Assembly (LEA). All of the secondary voltages are converted in the Power Converter Assembly (PCA) located in the magnesium box bolted directly to the spacecraft deck adjacent to the main MLA housing.

Command and data communications to the MESSENGER Payload Data Processing Unit (DPU) are through a low-voltage differential signal (LVDS) serial link using the RS-422 signal standard. An additional LVDS signal carrying the 5-MHz spacecraft reference clock is also provided to MLA and connects directly to the RMU board. The MLA instrument has single-string components, but can operate from either one of the redundant DPU, clock, and power connections. 
MLA has two defined software modes, Boot and Application. Boot mode is entered immediately after power up. MLA will remain in Boot mode until commanded to execute its onboard code residing in electrically-erasable programmable read-only memory (EEPROM), at which point it enters Application mode. Within Application mode there are three defined operational modes through which MLA will cycle every orbit. The lowest power mode is Keep Alive in which only the CPU, AEM and laser diode's thermo-electric cooler (TEC) are powered. Upon transition to Standby mode the RMU is powered on. Finally in Science mode the laser power supply is turned on and the laser fires.

\subsection{Technology Advances Implemented in MLA}

MLA has several new technologies, which provide a compact and efficient laser altimeter to the MESSENGER mission. The most prominent is the miniaturized laser transmitter shown in Figure 4, delivering $20 \mathrm{~mJ}$ of energy in a 70- $\mu \mathrm{rad}$ beam width from a compact $14 \mathrm{~cm} \times 9 \mathrm{~cm}$ package to facilitate high-resolution sampling of Mercury's surface. Its unique breathable filter allows for simpler testing in vacuum environments, providing significant test time in a flight-like environment.

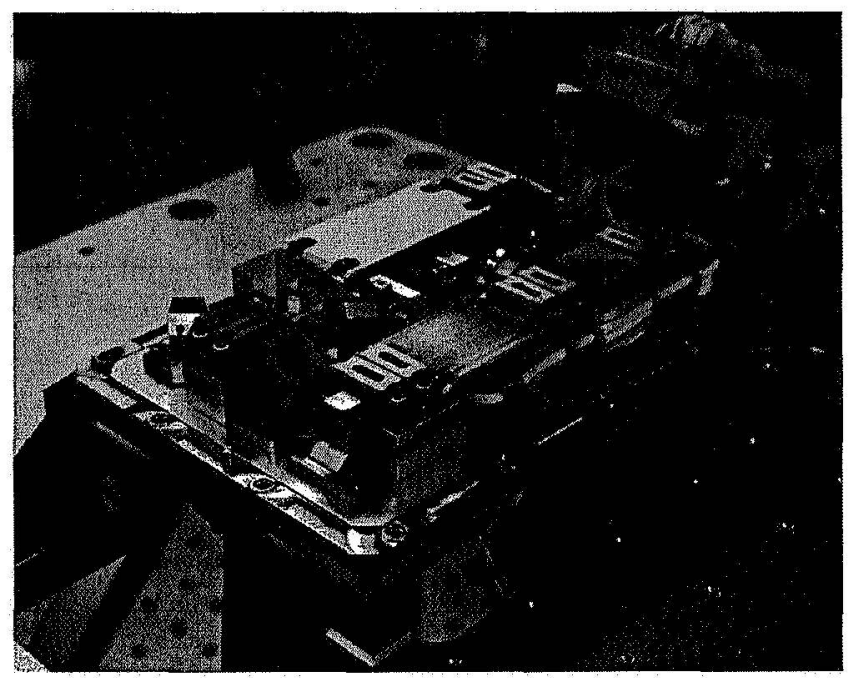

Figure 4 MLA Laser

MLA also incorporates a multiple aperture refractive telescope receiver, which has proven easier and less costly to manufacture and test than reflector telescopes used on previous altimeters. The modular design can be scaled to match the aperture requirements for a variety of missions. Fiber coupling makes the alignment and integration process simpler and decouples the detector assembly from the optomechanical integration and test (I\&T) flow.

The MLA Range Measurement Unit is another significant advancement, using a low speed $5-\mathrm{MHz}$ counter and time-of-flight ASIC developed by The Johns Hopkins University Applied Physics Laboratory (APL) to achieve timing resolution equivalent to a $2 \mathrm{GHz}$ counter with significantly less power. 
The detection scheme on MLA also makes possible operation and signal retrieval beyond the theoretical range limit of single-pulse detection electronics by acquiring and downlinking up to fifteen returns per shot. Correlation processing on the ground will be used to extract valid range signals from these additional data points.

\subsection{Laser Transmitter}

The MLA laser transmitter is an evolutionary design building on the past fifteen years of spaceflight laser designs from MOLA to GLAS. There are common features shared among these and other space-based lasers, which include a semiconductor laser-pumped solid-state approach to the laser, "zigzag" slabs made of neodymium-doped yttriumaluminum garnet that is chromium co-doped for radiation tolerance (Nd:Cr:YAG) with ends cut at Brewster's angle, and porro prism and mirror or crossed-porro prism laser resonators for stability against vibration and thermal stresses. The laser requirements are enumerated in Table 3. The total number of shots required to complete the mission is on the order of 30 million, a relatively small number when compared with other laser altimeter experiments such as MOLA-2 (660 million shots) and GLAS. Salient parameters such as pulse energy and pulse repetition rate are also lower.

\begin{tabular}{|l|l|}
\hline \multicolumn{1}{|c|}{ Parameter } & \multicolumn{1}{c|}{ Requirement } \\
\hline Wavelength & $1064.5 \mathrm{~nm} \pm 0.2 \mathrm{~nm}$ \\
\hline Pulse energy & $20 \mathrm{~mJ} \pm 2 \mathrm{~mJ}$ \\
\hline Pulse width & $6 \mathrm{~ns} \pm 2 \mathrm{~ns}$ \\
\hline Pulse repetition rate & $8 \mathrm{~Hz}$ \\
\hline Beam divergence $\left(1 / \mathrm{e}^{2}\right)$ & $801 \mathrm{rad}$ \\
\hline
\end{tabular}

\section{Table 3 MLA Laser Transmitter Requirements}

To meet these requirements the laser was implemented with an oscillator and amplifier design as shown in Figure 4 and Figure 5. The oscillator is a miniaturized version of the GLAS laser oscillator incorporating a zigzag Nd:Cr:YAG slab, GaInAsP laser diode pump arrays, a passive Q-switch, crossed porro prisms, and polarization output coupling. The laser diode arrays are temperature controlled with a TEC to maintain the optimum pump wavelength. The oscillator emits $3-\mathrm{mJ}$ pulses which are then fed through a $2 \mathrm{X}$ beam expander and a second Nd:Cr:YAG laser diode-pumped single-pass amplifier slab, which provides approximately 7 to $9 \mathrm{~dB}$ of amplification resulting in an output pulse energy of 15 to $22 \mathrm{~mJ}$. The gain of the amplifier stage is dependent on the temperature of the amplifier pump diodes and slab, which are not actively controlled (Krebs, et al., 2005). 


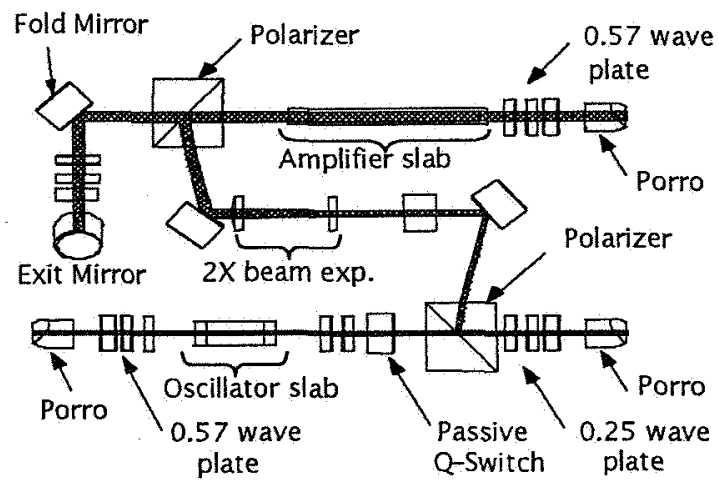

Figure 5 MLA Laser Optical Layout

The output of the amplifier stage is coupled into a $15 \mathrm{X}$ Galilean beam expander, which reduces the final beam divergence to approximately 70 microradians. Since the beam expander output face is exposed to the surface of Mercury during operations, a sapphire window is used to minimize IR coupling through its glass elements.

The MLA laser electronics assembly delivers a 100-A current pulse to the semiconductor laser diode arrays. The oscillator and amplifier diode arrays are connected in series and are driven from a capacitor bank charged to approximately 35 volts direct-current (VDC) generated as a regulated secondary supply by the MLA Power Converter. When triggered from the MLA timing electronics current is switched through the diode arrays by redundant metal-oxide-semiconductor field-effect transistors (MOSFETs). Current flows through the diode lasers until the oscillator Q-switch is bleached and a $1064 \mathrm{~nm}$ laser pulse is detected by a photodiode. The diode pump pulse is nominally $150 \mu \mathrm{s}$ in duration and is limited by the electronics to $255 \mu \mathrm{s}$. As the diode lasers age and emit less power the pump pulse will last longer allowing constant 1064-nm pulse energy output for the duration of the mission.

The laser electronics also implement a control loop to maintain the oscillator pump diode lasers at approximately $17^{\circ} \mathrm{C}$ via a thermoelectric cooler. The photodiode used to terminate the pump pulse is a quadrant detector that is placed behind a diffuser and views residual energy transmitted through the final turning mirror prior to the laser beam expander. Three segments of this detector are used for timing functions and the fourth segment output is used to monitor the energy.

One breadboard, one engineering model, and one flight model laser were fabricated at the Space Lidar Technology Center operated by the NASA Goddard Space Flight Center. Laser fabrication was performed in a Class-100 clean room. The engineering and flight units underwent vibration and thermal-vacuum cycling tests at the subassembly level during which all parameters were verified.

\subsection{MLA Receiver Optics}

The MLA receiver telescope design is a significant departure from prior telescopes used for MOLA, SLA, and GLAS. These altimeters all utilized Cassegrain reflector telescopes made with beryllium in the cases of MOLA and GLAS. The original design 
for MLA was in fact a beryllium reflector similar to these, but once the MESSENGER thermal environment was understood it became apparent that the Cassegrain reflector would not perform adequately with the significant thermal gradients resulting from viewing Mercury's surface. The requirements for the MLA receiver are listed in Table 4.

\begin{tabular}{|l|l|}
\hline \multicolumn{1}{|c|}{ Parameter } & \multicolumn{1}{c|}{ Requirement } \\
\hline Aperture & $417 \mathrm{~cm}^{2}$ \\
\hline Field of view & $400 \mathrm{rad}$ full angle, circular \\
\hline Bandpass filter & $0.7 \mathrm{~nm}$ FWHM pass band centered at $1064.5 \pm 0.2 \mathrm{~nm}$ \\
\hline Detector & $0.7 \mathrm{~mm}$ diameter silicon avalanche photodiode \\
\hline
\end{tabular}

\section{Table 4 MLA Receiver Optics Requirements}

The ensuing design shown in Figure 6 is a set of four refractive telescopes with an aperture equivalent to a single $0.25-\mathrm{m}$ diameter reflector with a $15 \%$ secondary obscuration. The objective lenses in each telescope are sapphire, chosen for its ability to withstand thermal shock, its lower absorption in the infrared and resistance to radiation darkening. At the back of each telescope is a dielectric fold mirror which reduces the total height of the assembly and reflects only a narrow band about $1064 \mathrm{~nm}$ allowing protection against accidental direct solar viewing by passing most visible solar radiation through its frosted back. At the focal plane of each telescope is a 200-mm core diameter fiber, which limits the field of view to $400 \mu \mathrm{rad}$. Alignment of each telescope's field of view (FOV) to the MLA laser is accomplished solely by translating each fiber at the focal plane (Ramos-Izquierdo et al., 2005).

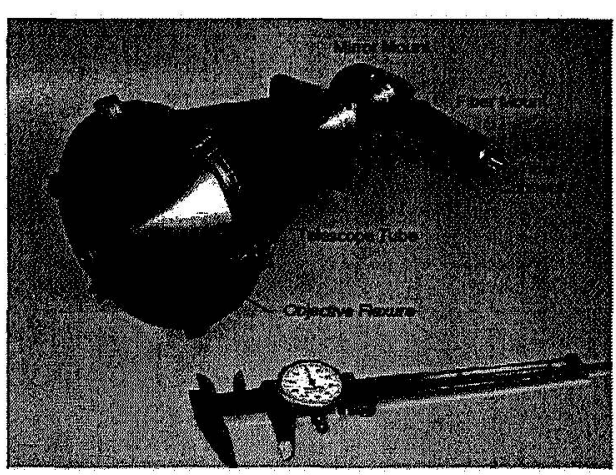

Figure 6 MLA Receiver Telescope

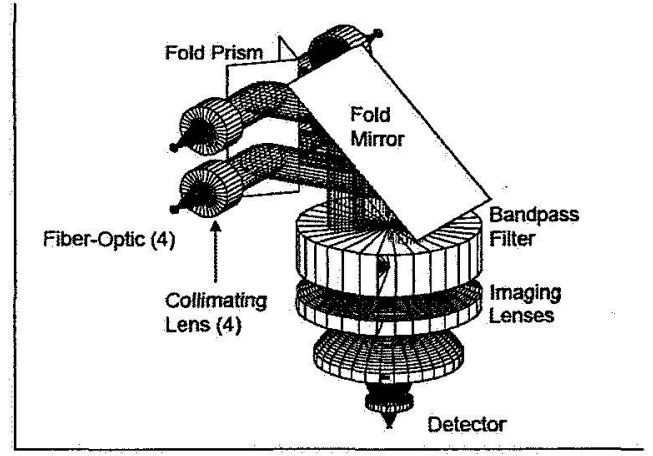

Figure 7 MLA Aft Optics

Each fiber optic couples the received power to a single aft optics assembly (Figure 7), which collimates the fiber outputs, and passes them through a single optical bandpass filter with a $0.7-\mathrm{nm}$ bandwidth. The center wavelength of the bandpass filter can be adjusted by tilting it to match the center wavelength of the laser pulse, which for MLA is $1064.5 \mathrm{~nm}$. After the bandpass filter the light from all four fibers is then focused onto a single silicon avalanche photodiode detector with a $0.7-\mathrm{mm}$ diameter.

Five engineering model (EM) telescopes and an EM aft optics assembly were fabricated out of aluminum to validate optical, mechanical, and thermal performance models. These 
EM units also served to develop the integration procedures and ground test equipment needed for the flight model telescopes. Four of the telescopes and the aft optics with fibers were also integrated with an aluminum MLA EM housing and laser beam expander assembly. This approach provided a complete optomechanical EM that also served to validate models and develop instrument-level alignment verification tests, which were used successfully on the MLA flight instrument.

\subsection{Receiver Electronics}

The detector hybrid, shown in Figure 8, was first developed during in the 1980s by then EG\&G Optoelectronics Canada for optical communication programs. It consists of a SiAPD chip, a low-noise preamplifier, and a high-voltage bias circuit, all contained in a hybrid circuit housed in a one-inch-diameter hermetically sealed package. These devices were used in SLA, MOLA-1, and MOLA-2, the latter of which has been operating in space since 1996 with little performance degradation. Additional devices with a wider electrical bandwidth and improved output pulse waveform fidelity were made for GLAS and used on MLA.

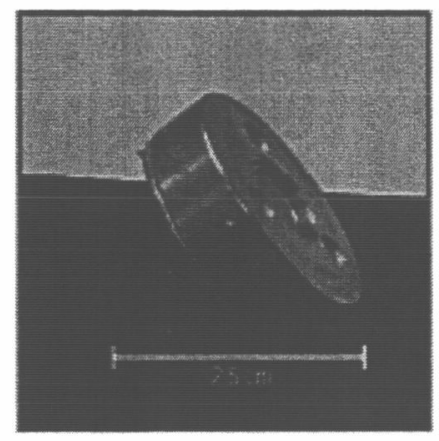

Figure 8 MLA Detector Hybrid

The electronics for received pulse detection duplicates in concept subsets of the circuits used for MOLA and GLAS and includes the same SiAPD detector hybrid and variable gain amplifier (VGA) stage as GLAS. The output of the VGA is split three ways that define the MLA "channels" 1 through 3. Channel 1 is the highest bandwidth version of the signal and has a 10-ns matched filter. The next two splits feed into the 60 ns and $270 \mathrm{~ns}$ matched filters. These latter two filter outputs are gain compensated for the filter loss. Channel 1 has two comparators, each with separately programmable thresholds (channel 1 "high" and channel 1 "low"). The remaining channels have single comparators. When the signal level crosses a programmed threshold voltage, the high-speed comparator switches a logic level, which is transmitted to the RMU via low-voltage differential signal (LVDS) interface for signal timing.

\subsection{Range Measurement Electronics}

Conceptually the MLA range timing circuitry builds on the basic pulse edge timing technique used for MOLA and SLA. The timing cycle for each laser pulse is shown in Figure 9. Rather than using the laser pulse emission to start the counter, as was done with MOLA, the trigger pulse to the laser is itself used as the start time and the laser pulse is the first event in each cycle. The important change is the implementation of a low- 
frequency counter operating at $5 \mathrm{MHz}$ coupled with a tapped delay line ASIC to determine the intra-cycle timing within the coarse counter resolution, providing much better resolution without the need for a high-frequency counter.

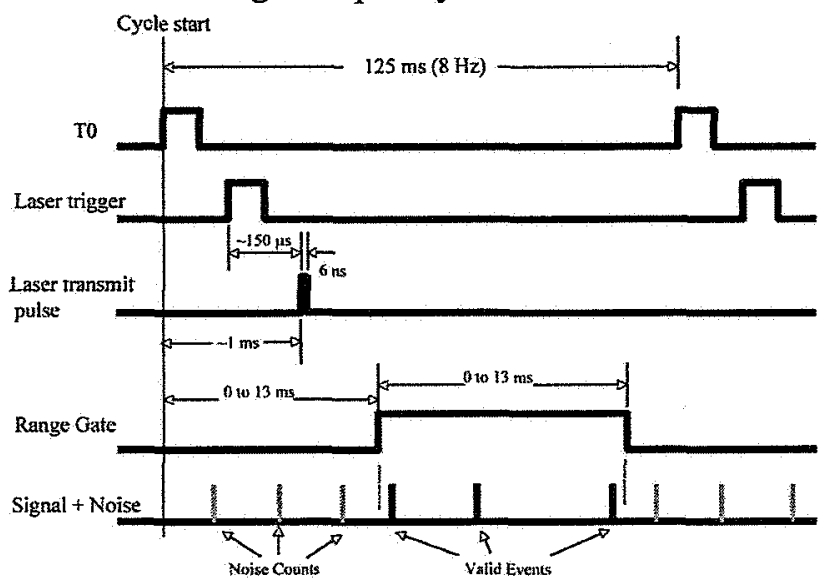

Figure 9 MLA Range Measurement Timing

The receiver event timers consist of a set of time-to-digital converters (TDC). The TDCs are based on the tapped delay line technique and each channel is implemented in a silicon ASIC specially designed for space applications, designated TOF-A by their JHU-APL developers (Paschalidis et al., 2002). The tapped delay lines consist of a series of logic gates with precise and uniform propagation delay times. An on-chip delay-lock-loop is used to self-calibrate the delay time against an external reference clock signal. The TOF-A can perform subnanosecond timing without the need for high frequency logic circuits and clock oscillators yet has limited full-scale range, thus a coarse resolution $5-\mathrm{MHz}$ counter is synchronized with each TOF-A to provide a full-scale pulse time-of-flight. Digital logic circuits are implemented in a field-programmable gate array (FPGA), an Actel RT54SX72S. The combined circuit can time the leading and trailing edges of the transmitted laser pulses and the received echo pulses to better than 500-ps accuracy with $13 \mathrm{~ms}$ dynamic range.

Six of these circuits comprise the MLA RMU. A signal edge designated T0 starts the coarse counters. Each edge of the LVDS comparator signals from the detector board starts a corresponding TOF-A and this circuit counts until the next leading edge of the 5-MHz clock stops it, providing sub-cycle timing. The start pulse and channel 1 "high" pulse TDCs record one event per edge (leading and trailing). The "low" threshold pulse TDCs can store multiple events from each shot, therefore a lockout circuit (as described in the overview) and a means to associate the source of the pulse (channel 1,2 or 3) are implemented. This circuit records the times of up to 15 events for every transmitted pulse with a dead-time of several hundred nanoseconds. A separate set of event counters totalizes pulses received on each channel inside the range gate. These counters are read and reset after every shot. 


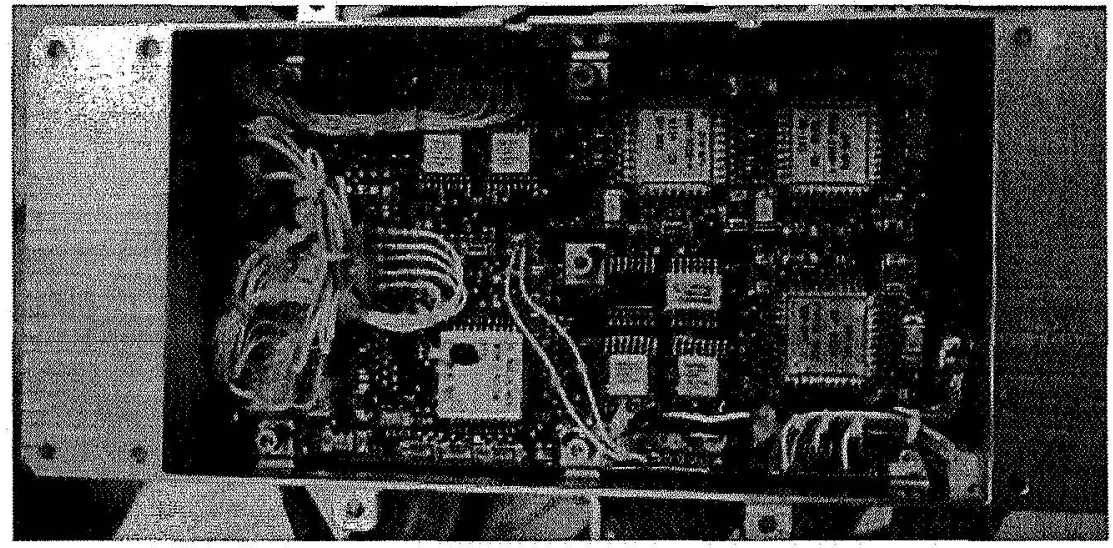

Figure 10 MLA RMU

The flight RMU is shown in Figure 10. An engineering unit is integrated into the instrument EM. Subassembly testing of the RMU demonstrated range precision capability under $1 \mathrm{~ns}$. Standard deviation of the error signal was approximately $250 \mathrm{ps}$ with a mean error of $480 \mathrm{ps}$.

\subsection{Command and Data Handling Electronics}

The MLA command and data handling $(\mathrm{C} \& D H)$ electronics are assembled on one printed wiring board referred to as the MLA CPU board. The unit was custom built for MLA but utilizes components with extensive heritage. The CPU itself is a 80CRH196KD device from United Technologies Microelectronics Corp. (UTMC), and is operated at a $16 \mathrm{MHz}$ clock frequency. All control and interface functions are implemented in an Actel RT54SX72S FPGA. Two each 64-kB programmable read-only memories (PROMs), 256$\mathrm{kB}$ EEPROMs, 512-kB static random-access memories (SRAMs), an oscillator and LVDS transceivers round out the board. A block diagram of the CPU is shown in Figure 11. The CPU board is shown in Figure 12.

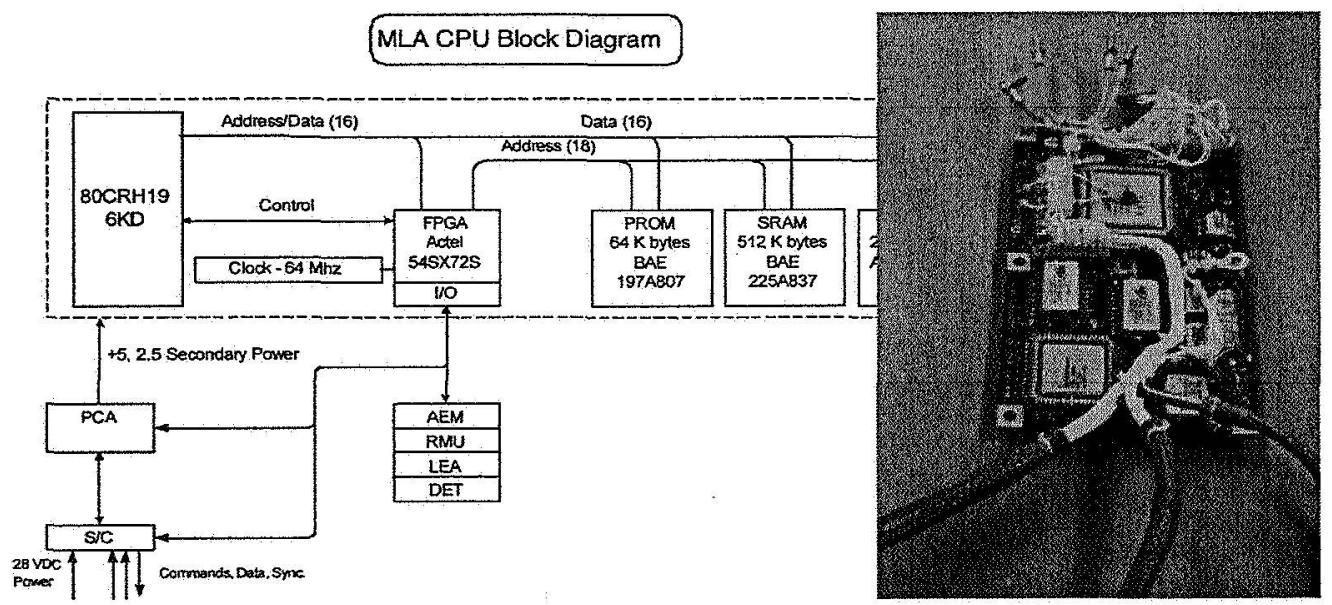

\section{Figure 11 MLA CPU Block Diagram}

Figure 12 MLA CPU Board

All command and data interfaces flow through the FPGA on the CPU board. Each MLA subsystem's control and data lines tie into this device. The FPGA therefore has a 
functional block for each MLA subsystem, two UART blocks for communications, one for address control and reset functions. The subsystem functional blocks are mapped to 80196 data memory space and defined for the Power Converter Assembly (PCA), Analog Electronics Module (AEM), and RMU. An interrupt controller is also implemented in the FPGA for interrupts from the RMU, AEM, address decode and the universal asynchronous receiver/transmitter (UART). All transfers to and from the subsystem and UART blocks are byte-wide only. Each UART is connected to a one of the redundant MESSENGER DPUs (A and B). All command and data transfer to the MESSENGER DPU passes through the UARTs. An additional UART integrated into the 80196 CPU was used for development. The 80196 timer function is also used to provide a reference to the phase of the spacecraft 1-pulse-per-second (1-PPS) signal and for flight software timing.

MLA operational modes (Keep Alive, Standby and Science, described in section 3.2) are defined by which subsystems are powered, so all mode changes are implemented by setting bits in the PCA block to enable secondary voltages. The RMU block transfers all range data and control functions. This block must function when the RMU is powered off as is the case in Keep Alive mode. The AEM block executes an autoconversion sequence to acquire laser energy and diode current monitor samples each time the laser fires without software intervention. All other analog-to-digital and digital-to-analog conversions are done under software control.

PROM memory contains the bootstrap code. One EEPROM device is permanently write protected and contains the last fully ground-tested version of the flight software. The second EEPROM can be overwritten via ground commands to allow updates to the flight software during the mission.

One breadboard, two engineering models, and one flight unit were fabricated for MLA. The breadboard CPU was utilized for flight software development and testing, and one EM was used in the instrument EM.

\subsection{Power Converter Electronics}

The same designer who executed the power converters for MOLA and GLAS developed the MLA Power Converter Assembly (PCA). Building upon this experience MLA has smaller and more efficient converters. The MLA PCA is shown in Figure 13. 


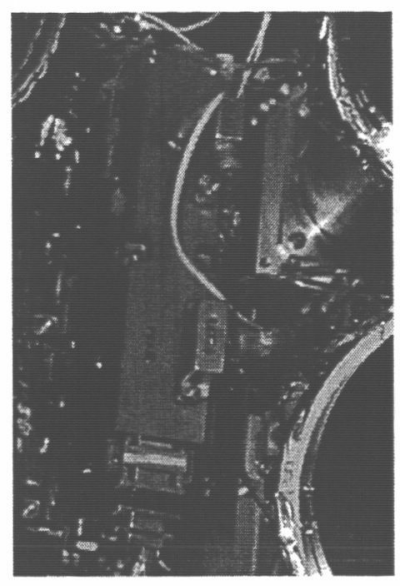

Figure 13 MLA Power Converter Assembly

The PCA generates all the secondary voltages on MLA. Requirements for secondary voltages and the subsystems that use them are enumerated in Table 5 . The $2.5-\mathrm{V}, 35-\mathrm{V}$ and 550-V converter outputs are all switched on or off by control bits from the CPU FPGA to control power consumed by MLA. The $12-\mathrm{V}, 5-\mathrm{V}$ and $2.5-\mathrm{V}$ sources are forward converter topology DC-DC converters. The negative 5-V source is produced from the positive $5-\mathrm{V}$ supply by a flyback converter. The 550-V SiAPD bias voltage is also converted from the positive 5-V supply by a resonant converter. A $35-\mathrm{V}$ source used solely for charging the capacitor bank that powers the laser pump diodes is implemented in a flyback converter.

\begin{tabular}{|c|c|c|c|c|c|c|}
\hline \multirow[t]{2}{*}{ Subsystem } & \multicolumn{6}{|c|}{ Power required (W) } \\
\hline & $+2.5 \mathrm{~V}$ & $+5.0 \mathrm{~V}$ & $-5.0 \mathrm{~V}$ & $+12.0 \mathrm{~V}$ & $35 \mathrm{~V}$ & $+550 \mathrm{~V}$ \\
\hline CPU & 0.275 & 0.87 & 0 & 0 & 0 & 0 \\
\hline RMU & 0.088 & 0.25 & 0 & 0 & 0 & 0 \\
\hline RMU heater & 2 & & & & & \\
\hline Detector & 0 & 0.775 & 0.47 & 0 & 0 & 0.01 \\
\hline Analog board & 0 & 0.157 & 0.073 & 0.019 & 0 & 0 \\
\hline Laser electronics & 0 & 0.26 & 0.11 & 0.324 & 5.54 & 0 \\
\hline Laser thermo-electric cooler & 0 & 2.5 & 0 & 0 & 0 & 0 \\
\hline Laser amplifier heater & 0 & 0 & 0 & 2 & 0 & 0 \\
\hline Load totals & 2.36 & 4.81 & 0.65 & 2.34 & 5.44 & 0.01 \\
\hline Power converter dissipation & 1.11 & 1.44 & 0.35 & 0.91 & 0.74 & 0.20 \\
\hline Power requirement & & & & 20.37 & & \\
\hline Schottky diode loss & & & & 0.370 & & \\
\hline Average prime power requirec & & & & 20.75 & & \\
\hline
\end{tabular}

Table 5 PCA Power Requirements

The laser, operating at an $8-\mathrm{Hz}$ pulse rate, discharges the capacitor bank by switching 100 amps through the laser pump diodes in a 150 - $\mu$ s current pulse. The capacitor bank must then be recharged in time for the next pulse. The resultant charge current, drawn through the $35-\mathrm{V}$ converter is reflected on the spacecraft power bus current in the form of an $8 \mathrm{~Hz}$ 
square wave with approximately $700-\mathrm{mA}$ amplitude and duty cycle from $25 \%$ at maximum bus voltage to $80 \%$ at minimum bus voltage as shown in Figure 14 . Since the MESSENGER general electromagnetic compatibility (EMC) requirements specified that current ripple be less than $500 \mathrm{~mA}$, a waiver was granted for MLA allowing up to $800-\mathrm{mA}$ ripple. Subsequent cross-compatibility tests showed that this ripple did not affect other spacecraft subsystems.

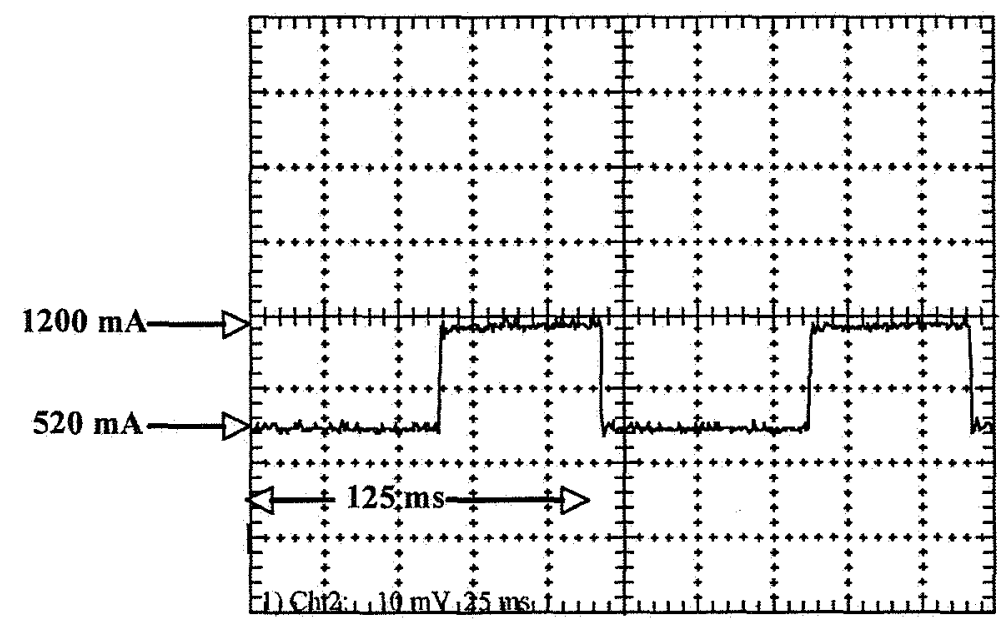

Figure 14 MLA PCA Current

The $8-\mathrm{Hz}$ laser trigger is synchronized to the spacecraft $1-\mathrm{Hz}$ timing reference. Since the spacecraft C\&DH samples the power bus current synchronously with this reference the MLA current is always sampled at the peak of $8-\mathrm{Hz}$ square wave described above.

The PCA is housed in a magnesium box, which is bolted directly to the spacecraft deck. A thermal gasket allows conduction of dissipated power to the deck. The spacecraft power and communications connectors are on the PCA box. Command and data signals are simply routed through the PCA to the MLA CPU board.

One engineering model and one flight model PCA subassembly were delivered to GSFC by Spacepower, Inc., the contractor for the PCA design and fabrication. The units as delivered were functionally tested over the specified temperature range.

\subsection{Software}

The MLA flight software consists of multiple tasks running under a real time executive. The two main groups of tasks are the C\&DH set comprised of timing, communications, system maintenance, and the Science tasks that acquire, process, and compress the critical science data. Code for MLA was developed in the $\mathrm{C}$ programming language and operates under a Real Time Operating System (RTOS) from CMX Systems. The MLA Flight Software Tasks are depicted in Figure 15. 


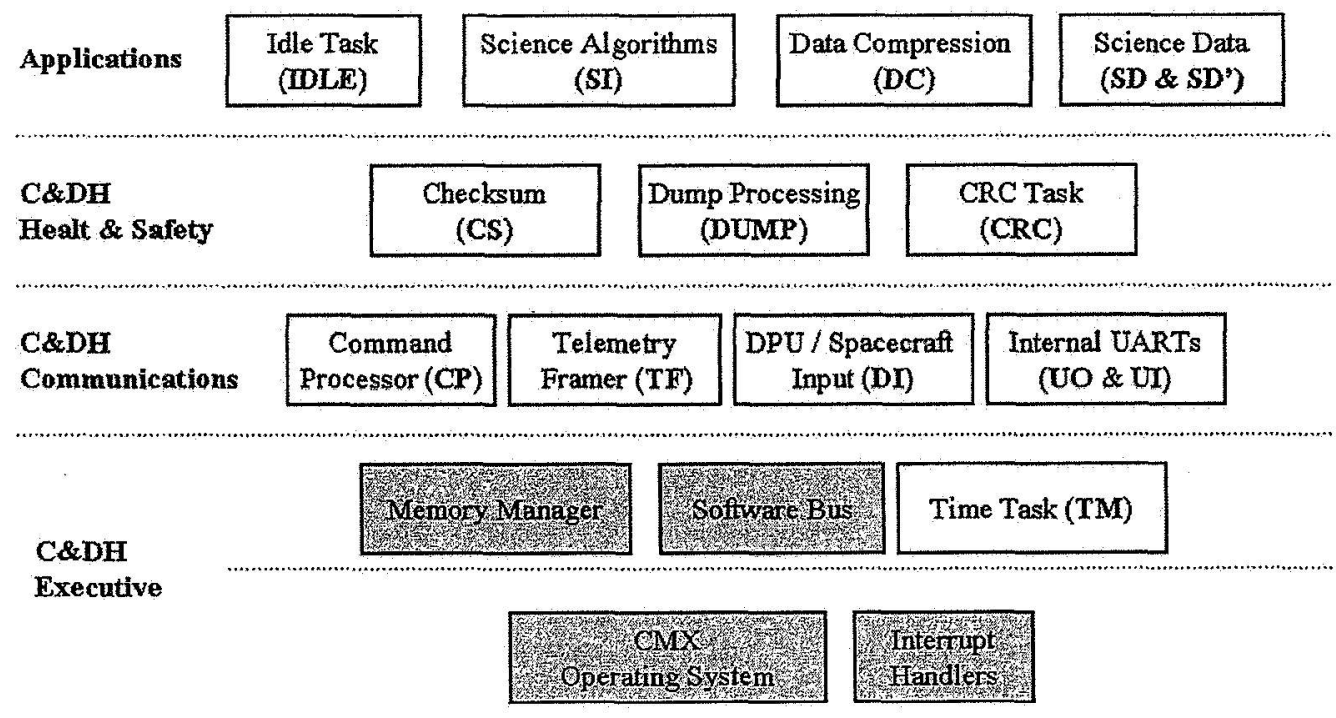

Figure 15 MLA Flight Software Tasks

The science algorithm builds on heritage primarily from MOLA and Microaltimeter experience. Like the MOLA software, the MLA algorithm integrates background noise counts (detector events outside the range gate) and sets comparator thresholds to minimize false detections within the range window. MLA also utilizes line-of-sight range information from the spacecraft attitude control system (ACS) to update the range gate delay, width, and detector gain based on altitude and descent or ascent rate. More significantly the MLA algorithm takes in the multiple returns from the RMU low channel and maintains a histogram of range measurements within the window. If a valid return is detected on the high threshold channel then the three low returns closest to it are downlinked to the ground. If there is no high channel return then all the low returns (up to a preset limit) are downlinked to the ground to be processed using correlation techniques.

The flight software timing is handled by the RTOS and interrupt service routines (ISR). The RTOS is a pre-emptive priority-based scheduler with semaphore and delay functionality. The ISRs are immediately branched to by the processor when their associated interrupt is generated, except when they are masked off, in which case the interrupt is pending, but no action by the processor is taken.

The base cycle period of the flight software is one second. The spacecraft demarcates this period with the 1-PPS signal. All telemetry for that second is required to be packaged in an Instrument Transfer Frame (ITF) and sent to the spacecraft. Each second the flight software runs an entire pass through all of its code. There are some tasks that have counters that enable them to do different things on different seconds, but those counters are always maintained locally to the task.

There are three scheduling sequences that occur within the second. The first is the internal flight software timing sequence, the second is the RMU laser firing sequence, and the third is the purely data-driven low-priority tasks that are scheduled either from command packets or the time message. Both of the first two sequences are naturally asynchronous, but both 
of them are configured to synchronize with the 1-PPS and therefore, in effect, synchronize with each other. The last sequence is left asynchronous.

When the MLA instrument is powered on, the power-on reset state of the software is the Boot Loader code. Every time the CPU board is powered on or reset, the Boot Loader executes. From the Boot Loader, a command is required to signal to the software that the Boot Loader should load the flight software out of EEPROM, into SRAM and jump execution to it.

There are three ways to reset the MLA instrument: a processor reset, a FPGA watchdog timeout, and a spacecraft power cycle. All three ways can be commanded. There is, on the other hand, only one way to go from the PROM Boot Loader code to the EEPROM flight software code, and this is by processing a load directive. A load directive is a structure in memory used by the Boot Loader that contains instructions on where and how to initialize SRAM, as well as where to copy sections of EEPROM code and data into SRAM to be executed. There are two commands that cause the Boot Loader to process a load directive.

Bench testing was performed with the MLA flight software executing on a PC-based simulator, the MLA CPU breadboard, and finally on the MLA EM prior to loading into the MLA flight instrument EEPROM. Numerous full orbit simulations with the spacecraft also provided valuable performance data.

\subsection{Mechanical Design}

MLA's structural elements, like MOLA and GLAS, are made primarily of beryllium. The PCA box is magnesium to minimize mass. A significant departure from previous altimeters is the use of four refractive telescopes, also made with beryllium. The main mechanical elements of MLA are depicted in Figure 16.

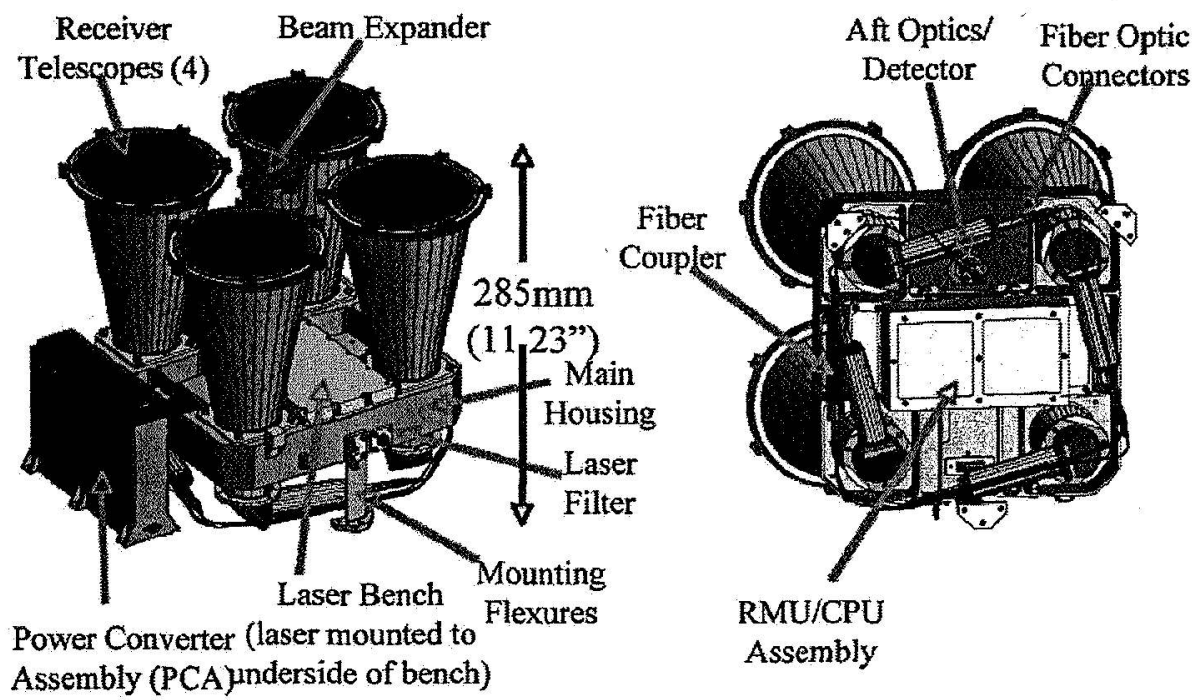

Figure 16 MLA Mechanical Subassemblies 
The primary metering structure that maintains the boresight alignment is the MLA main housing. This beryllium structure supports the four telescope tubes, the beam expander, and laser bench. The detector, aft optics, and housekeeping electronics also occupy the housing, and a separate box with the RMU and CPU is bolted to the housing. The main challenge in the design of this structure is to keep the telescope tubes co-aligned with the beam expander over the extreme temperature swings to be seen on orbit. Tight assembly tolerances were required to bring all four telescope tubes to within 2 mrad of the beam expander output at initial assembly. The fiber optic couplers could then be adjusted to align the receiver and transmitters to within $10 \mu \mathrm{rad}$. The main housing bolts to the spacecraft deck with three titanium flexures to minimize thermal conduction to the deck and avoid mechanical distortion of the housing.

The laser bench is a $14-\mathrm{cm}$ by $9.3-\mathrm{cm}$ slab of beryllium to which the laser resonator and amplifier components are mounted. The beam expander bolts directly to this bench and comprises the laser subassembly. The housing has a cavity to contain the laser, which was precision cleaned and kept sealed until laser integration. This cavity has a breathable filter that allows the laser to vent during vacuum testing and after launch. The laser bench is bolted to the housing with 14 bolts around the perimeter. The four beryllium telescope tubes also bolt directly to the housing with four bolts each. The lens mounts for the beam expander and telescope tubes are titanium and hold the BK7G18 glass optics for the beam expander and the sapphire objective lenses in the telescopes.

The main housing, laser bench, and electronics housing were all gold plated for thermal performance. Most of the beryllium used is instrument grade (I-220-F) save for the telescope flanges, which are structural grade. All beryllium parts are nickel plated to prevent oxidation and personnel exposure. Phosphor-bronze helicoils were utilized for all bolt holes. Aluminum engineering models of all components were fabricated to develop assembly and integration procedures and to validate structural models.

\subsection{Thermal}

MLA will operate under a harsh and highly dynamic thermal environment due to the large variation in heat flux from the Mercury surface from daytime, nighttime, and deep space views. The transmitter and receiver optics undergo a rapid and uneven temperature rise during the science measurement at a rate of tens of degrees per hour in the laserbeam expander and the receiver telescope.

MLA acquires data for 15 to 45 minutes each orbit during which it uses the maximum amount of power available. Data acquisition takes place near periapsis, where radiative input from Mercury's surface is at a maximum. These factors result in a significant temperature rise through the instrument during the data pass. The remaining 11 hours of the orbit are used to radiate the absorbed heat into deep space. The primary radiators are the telescopes tubes and the laser beam expander. MLA, as with the rest of the spacecraft, never reaches thermal equilibrium during operations and must undergo $\sim 700$ thermal cycles during the mission. Figure 17 shows the temperature changes typically seen during an orbit. 



Figure 17 MLA Thermal Predicts (values in ${ }^{\circ} \mathrm{C}$ )

The entire instrument is covered with a 10-layer multi-layer insulation (MLI) blanket as shown in Figure 18. The outer layer of the blanket is vapor deposited gold (VDG) and the inner layers are vapor deposited aluminum (VDA). During spacecraft I\&T a layer of aluminized Kapton was added to the nadir-facing surface to alleviate concerns about solar illumination of the VDG layer. A thin strip of black Kapton was also added to minimize reflections on the side facing the MESSENGER Dual Imaging System, MDIS (Hawkins et al., 2005). The PCA box conducts its dissipated power directly to the spacecraft deck and is enclosed in another MLI blanket to avoid radiative coupling to the rest of the instrument. Titanium flexures minimize conduction from the main housing to the deck and another MLI blanket underneath the instrument isolates it from the spacecraft. The sapphire objective lenses have excellent thermal conductivity compared with other transparent materials and while they absorbs about half of the infrared radiation from the planet's surface they can withstand the resultant thermal shock. A sapphire flat is also used on the beam expander output to improve its thermal properties.

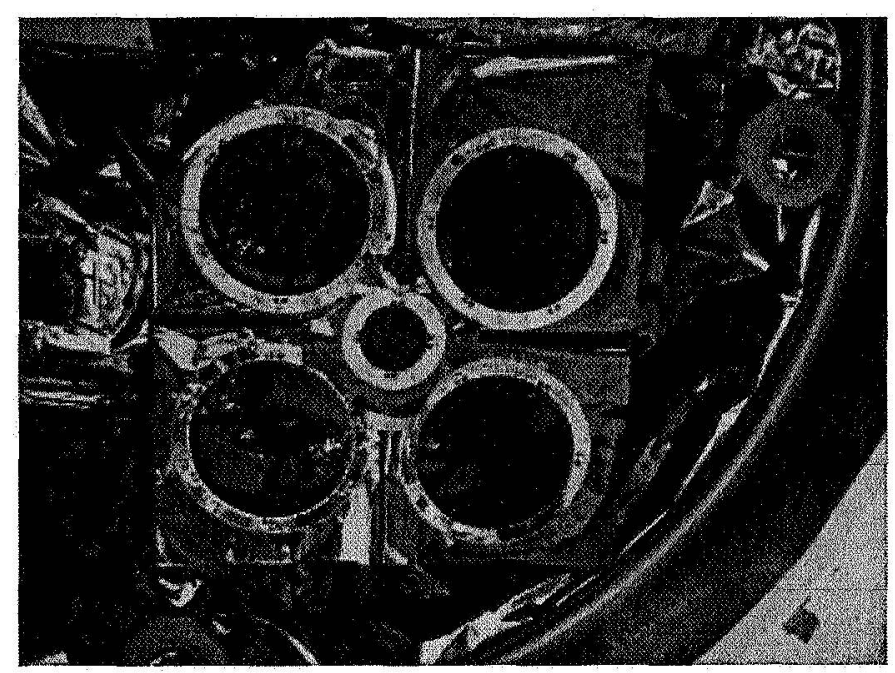

Figure $18 \mathrm{MLA}$ with thermal blankets 
Two sets of 17-W survival heaters, powered by separate spacecraft circuits are used. The " $\mathrm{B}$ " side circuit has a $-20^{\circ} \mathrm{C}$ thermostat and the " $\mathrm{A}$ " side trips at $-15^{\circ} \mathrm{C}$. During operations the " $A$ "-side heaters will be used. The " $B$ "-side heaters are needed during cruise and on orbit eclipse cases.

Laser transmitter performance is dependent on the laser bench temperature. Optimal operating temperatures for the laser bench range from $15^{\circ} \mathrm{C}$ to $25^{\circ} \mathrm{C}$, so to maximize laser energy during the data acquisition part of the orbit the laser bench should be at $15^{\circ} \mathrm{C}$ just prior to entering Science mode. To achieve this objective an operational heater is located on the laser bench and is powered by the 12-V supply when MLA enters Standby mode. The duration in Standby mode may be modulated through the mission to achieve this starting temperature under varying orbit conditions. Currently the predicted time in Standby mode ranges from 15 minutes for the hot case to 480 minutes for the cold case.

\section{MLA System Integration and Test}

\subsection{Instrument Assembly}

The MLA assembly sequence was driven primarily by the cleanliness requirements for the laser. Prior to any assembly activity the main housing was precision cleaned, and the cavity that enclosed the laser was sealed during integration of the electronics with the housing. Laser bench integration was performed in a Class 100 clean room. Once the laser was sealed in the housing a ground-support equipment (GSE) air filter was attached in series with the built-in flight filter to provide additional protection during I\&T.

The receiver telescope tubes and aft-optics were assembled on a Class 100 laminar flow bench in a Class 1000 clean room. The MLA housing with the laser and electronics was delivered to this area, and the telescope tubes were attached. The assembled instrument was then attached to its handling fixture. Before the fiber optics were connected a series of free-space tests with the laser were performed to establish baseline performance and to calibrate the GSE power measurements which would be used throughout ground testing to monitor laser performance.

MLA was then mounted to an optical bench that contained the alignment optics for boresighting. The optical axis of the alignment fixture was co-aligned with the MLA laser's optical axis. Each receiver's fiber optic was then aligned to the fixture's axis. As a final check each fiber was back-illuminated while the laser was firing, producing an image at the alignment fixture's focal plane showing the laser image with the receiver's illuminated FOV. Since the alignment was done in the lab at one atmosphere, shims on the fiber optic connectors positioned each fiber at the vacuum focus of each telescope. The fibers were then attached to the aft optics, completing the assembly sequence prior to instrument level testing.

\subsection{Contamination}

After assembly of MLA the particulate contamination requirements were relaxed to Class 10000 level since the most sensitive optics were sealed. All optical surfaces, particularly the beam expander output window, were visually inspected prior to firing the laser. MLA GSE that came in close contact with the instrument was cleaned and inspected prior to 
each use. GSE covers were made for each telescope objective and the beam expander window. These were attached when the instrument was not under test. After MLA was installed on the spacecraft with the thermal blankets, a sheet of lumalloy was taped over the receiver objectives when not testing. Final inspection and cleaning of all optical surfaces was performed prior to encapsulation using bright white and ultraviolet light sources.

The GSE air filter remained attached to the laser housing until final closeout at the spacecraft level save for mass properties testing during which a small plug was installed. A gaseous nitrogen purge was applied to the spacecraft payload area during integration up to launch. This purge line was filtered and a manifold distributed the purge gas to each instrument. The purge line for MLA was attached near the laser filter snout at final closeout after the GSE air filter was removed.

\subsection{Performance Testing}

Tables $6 \& 7$ show the MLA ranging and optical alignment error budgets associated with the performance requirements listed in Table 1. A suite of tests was designed to verify MLA performance at the instrument level and during spacecraft testing. Subsets of this suite were used for Aliveness and Functional testing, and the entire suite comprised the Comprehensive Performance Test. Ancillary tests such as boresight alignment verification and timing tests were used at key points during Integration and Test as calibration points and to demonstrate compliance with the MESSENGER Component Environmental Specification.

\begin{tabular}{|l|c|}
\hline \multicolumn{1}{|c|}{ Error Source } & Contribution \\
\hline Leading edge timing & $0.06 \mathrm{~m}$ \\
\hline Clock frequency error $(0.1$ parts per million & $0.20 \mathrm{~m}$ \\
\hline Measurement quantization $(2.5 \mathrm{~ns})$ & $0.11 \mathrm{~m}$ \\
\hline Pointing angle uncertainty $(0.13 \mathrm{mrad})$ & $0.68 \mathrm{~m}$ \\
\hline Spacecraft orbit knowledge error & $0.75 \mathrm{~m}$ \\
\hline Total (rss) & $1 \mathrm{~m}$ \\
\hline
\end{tabular}

Table 6 MLA Single Shot Ranging Margins

\begin{tabular}{|l|c|}
\hline MI. A In tegration Margins & Specification \\
\hline Laser beam axis parallel to receiver telescope axis & $<2 \mathrm{mrad}$ \\
\hline Laser beam axis perpendicular to mounting plane & $<5 \mathrm{mrad}$ \\
\hline MLA Alignment Margins & \\
\hline Receiver telescopes to laser beam axis (boresight) & $+/-50 \uparrow \mathrm{rad}$ \\
\hline Laser beam axis to MLA reference cube (knowledge) & $+/-50 \mu \mathrm{rad}$ \\
\hline MIA Stability & \\
\hline Laser beam axis to MLA mounting plane & $+/-50 \uparrow \mathrm{rad}$ \\
\hline Receiver telescopes to laser beam axis (boresight) & $+/-100 \uparrow \mathrm{rad}$ \\
\hline
\end{tabular}

Table 7 MLA Optical Alignment Margins 


\subsubsection{Bench Checkout Equipment}

The set of test equipment used to verify performance was in itself a system of electronic, electro-optic, and optomechanical subsystems referred to as the Bench Checkout Equipment (BCE). Referring to the Figure 19, the BCE consisted of three main pieces. One equipment rack containing the main data acquisition system, spacecraft interface simulator, and timing and electro-optic sources, one rack containing the alignment data acquisition and control system, and an optomechanical target assembly used for functional and alignment testing. In addition several hundred meters of fiber optics were utilized to couple signals and provide constant time-delay paths.

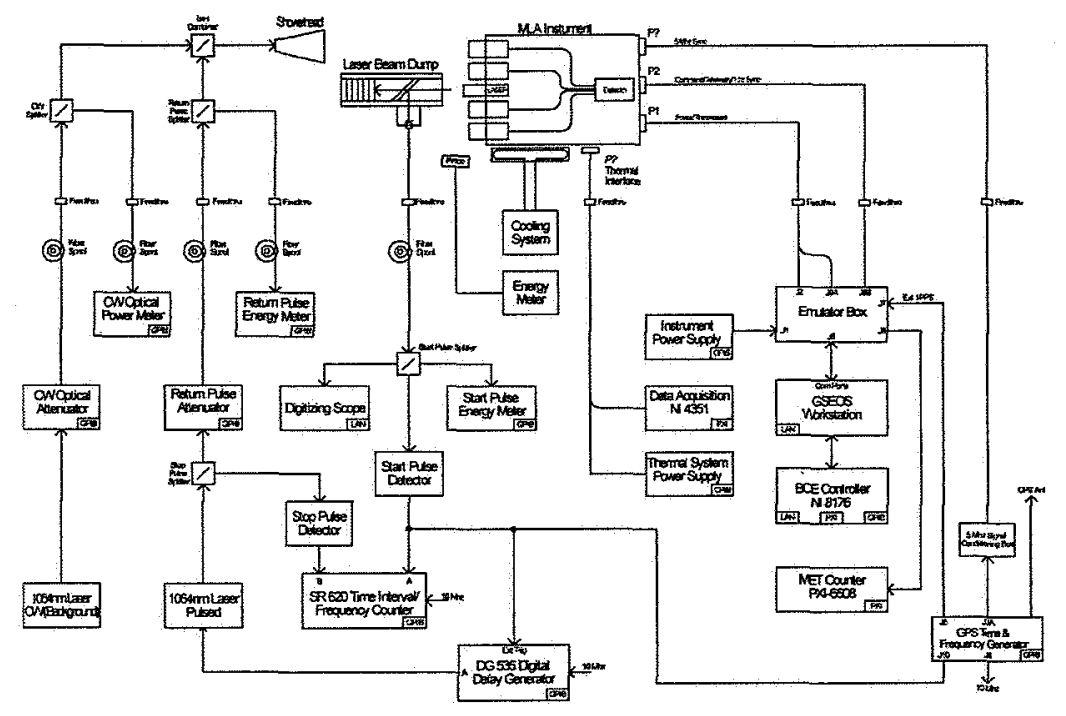

Figure 19 MLA BCE Functional Block Diagram

During instrument-level testing the BCE utilized the MESSENGER-supplied spacecraft interface simulator to provide power and transfer commands and data to and from MLA. After integration with the spacecraft the $\mathrm{BCE}$ acquired real-time telemetry via the MESSENGER ground data system network. During all phases of testing the BCE captured and time-stamped each and every laser pulse emitted by MLA and provided simulated return signals and noise at the MLA receiver telescope. Laser pulse energy was continuously monitored.

Simulated return signals were generated by a diode-pumped Nd:YAG laser housed within the rack that produced 5- $\mu \mathrm{J} 1-\mathrm{ns}$ pulses when triggered by the timing electronics. These pulses were fed through an optical attenuator and fiber coupled to a holographic diffuser, which distributed the signal power in a 0.006 -steradian cone to be collected by one of the MLA receiver telescope apertures. Optical background noise was simultaneously coupled in a similar manner using a continuous-wave (CW) $\mathrm{Nd}$ :YAG laser source.

The BCE used a Stanford Research Systems SR620 time-interval analyzer and two InGaAs photodiodes to independently measure the delay between each emitted MLA laser pulse and the corresponding simulated return pulse. The timebase for the SR620 is a rubidium-based oscillator. The drift of this oscillator is measured by recording the 
phase between its $1-\mathrm{Hz}$ output to a $1-\mathrm{Hz}$ tick generated by a Global Positioning System (GPS) receiver.

\subsubsection{Laser Performance Testing}

Two different laser beam termination schemes were utilized for monitoring the MLA laser. Both were designed to provide a "light-tight" seal to the MLA beam expander to prevent damaging levels of power from leaking into the receivers and afford an additional level of personnel safety. In addition both schemes were required to minimize back reflections into the laser itself.

One assembly is illustrated in Figure 20. This scheme, known as the beam dump, was an integral part of the alignment test fixture and used a beam splitter to direct $90 \%$ of the laser pulse power into a holographic diffuser and then focused a portion thereof into a 0.22 numerical-aperture optical fiber for transmission to a Molectron JD2000 Joulemeter energy monitor and an InGaAs photodiode used for timing and temporal pulse width measurement. The remainder of the energy was transmitted through to a charge-coupled device (CCD) camera used for beam diagnostics and redirected via retroreflector into one of the four MLA telescopes. Filter holders allowed for placement of volume absorbing neutral-density filters to attenuate optical power to usable levels.

The second assembly, referred to as the beam stop, was used primarily for spacecraftlevel testing and incorporated a Macor ceramic insert to diffusely reflect the incident laser power. Depending on thickness, Macor transmits some power at $1064 \mathrm{~nm}$ allowing for placement of an optical fiber on the back of the beam stop used with the BCE in the same manner as the beam dump fiber.

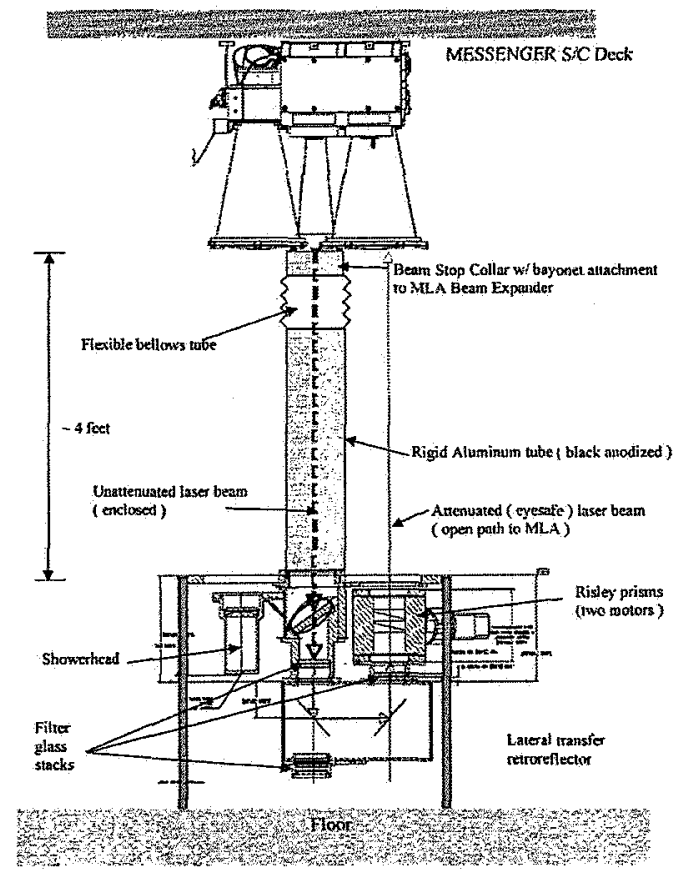

Figure 20 MLA Laser Beam Dump and Alignment Fixture 


\subsubsection{Boresight Alignment Verification}

Two different methods were used to verify the MLA boresight alignment, or angular offset between the transmitted laser beam and each receiver telescope's field of view.

The initial boresight alignment was performed on a laboratory optical bench using a 2.5$\mathrm{m}$ focal-length collimator with a $400-\mathrm{mm}$ diameter off-axis parabola. The initial alignment described in section 4.2 used this fixture. To verify the alignment with this fixture the MLA laser beam axis was aligned to the optical axis of the fixture. With the MLA laser turned off, a 1064-nm CW point source at the focus of the fixture was mechanically swept across the MLA field of view while monitoring noise counts in MLA telemetry. MLA noise counts were then plotted against the point source offset to assess the receiver alignment. This method also enabled simultaneous alignment testing of all four receiver telescopes.

During MLA instrument environmental testing and spacecraft-level testing the laser beam alignment to the receiver telescopes was measured by redirecting a portion of the transmitted laser pulse back into one of the four receiver tubes using a lateral transfer hollow retroreflector (LTHR). At the output of the LTHR a pair of motorized Risley prisms deflected the beam at a programmable angular offset into the telescope. By sweeping the angular offset in orthogonal directions across each telescope's field of view and monitoring the power received at the MLA detector, a centroid was computed that indicated the relative angular offset of the laser beam to each telescope. Relative pulse power received was measured by computing the change in detected pulse width and normalizing for each tube. This setup is depicted in Figure 20.

\subsection{Calibration and Characterization}

\subsubsection{Instrument Calibration}

MLA provides three measurements: the laser-pulse time-of-flight, the echo-pulse width, and the echo-pulse energy, along with a precise epoch time. To calibrate these measurements the MLA BCE continuously monitored laser pulse energy and range timing over the entire dynamic range of the instrument at all detector gain settings and comparator thresholds. Each simulated range signal was simultaneously measured with a calibrated time interval analyzer. Pulse propagation through several different fixedlength optical fibers was also used to calibrate the range measurement.

To locate the measurement point on Mercury's surface a precise knowledge of the MLA boresight axis relative to the spacecraft frame is also needed. Pointing verification tests were performed during instrument thermal-vaccum (TVAC) testing, and on the spacecraft after integration, during TVAC, and prior to launch at the Astrotech integration facility in Titusville, Florida.

Timing tests with the MESSENGER spacecraft were also performed after integration to verify that each laser shot was correctly time-stamped. All timing measurements were referenced to GPS-based Universal Time Coordinated (UTC) epoch time. 


\subsubsection{Environmental Testing}

All engineering models of electronic, optical, and laser subassemblies were thermally cycled at ambient pressure. Optics were tested under vacuum to verify focus shift. The EM and flight laser subassemblies both underwent full thermal vacuum testing while monitoring beam quality, pulse shape and energy.

A planet simulator target was also used during instrument-level thermal balance testing to provide a radiative input to MLA and measure the instrument's response to thermal transients. These test provided valuable insight to the thermal performance under onorbit conditions. The MLA thermal vacuum (TVAC) test setup is shown in Figure 21. Cold plates were attached to the laser bench and main housing to achieve qualification temperatures.
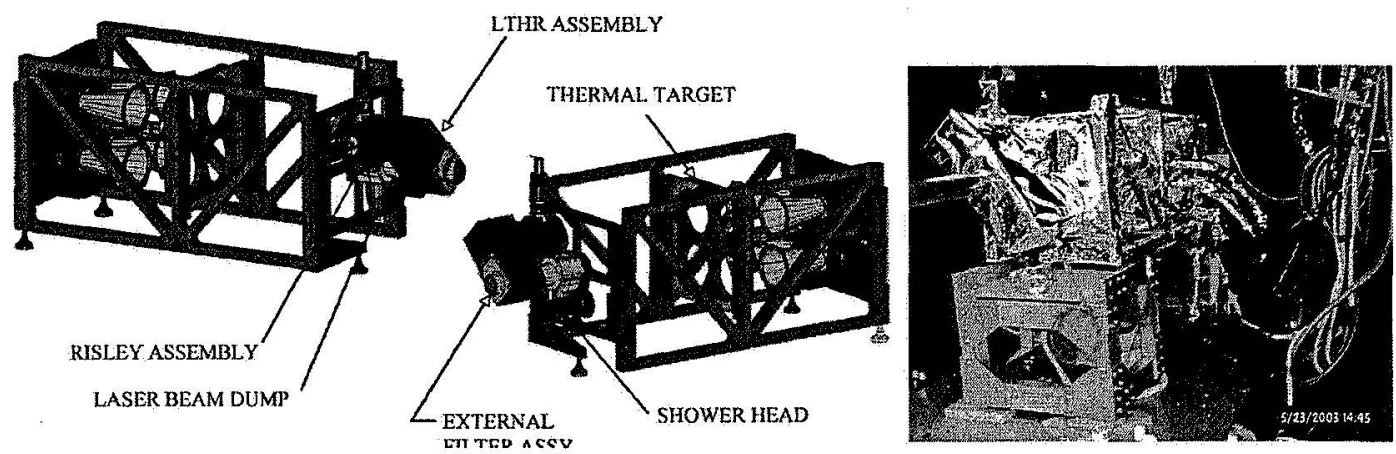

Figure 21 MLA TVAC Test Fixture

During instrument TVAC testing the laser pointing was continuously monitored using a telescope and CCD camera outside the chamber that imaged the MLA laser spot along with a reference cube on the instrument. Boresight verification was performed using the LTHR and Risley setup at each TVAC test plateau as well. After instrument TVAC testing the boresight was again verified using the optical bench setup.

During spacecraft environmental testing cold plates were again attached to the MLA laser bench and main housing to provide additional temperature cycles. MLA testing on the spacecraft was limited to range timing with simulated signals. Pointing verification was done before and after spacecraft TVAC.

\subsection{Post Launch Checkout Results}

MLA was first powered on 19 August, 2004, 16 days after launch $(\mathrm{L}+16)$. It remained in Standby mode for approximately 24 hours to allow time for the laser bench to warm up. This warm up also allowed any contaminants (our primary concern was water condensate left as ice on the optics) to sublime. A detector noise characterization test was performed shortly after turn on and the results are shown in Figure 22 along with equivalent TVAC test results. Detector noise appeared nominal on all channels. Another noise characterization was run on $\mathrm{L}+17$ with similar result. 


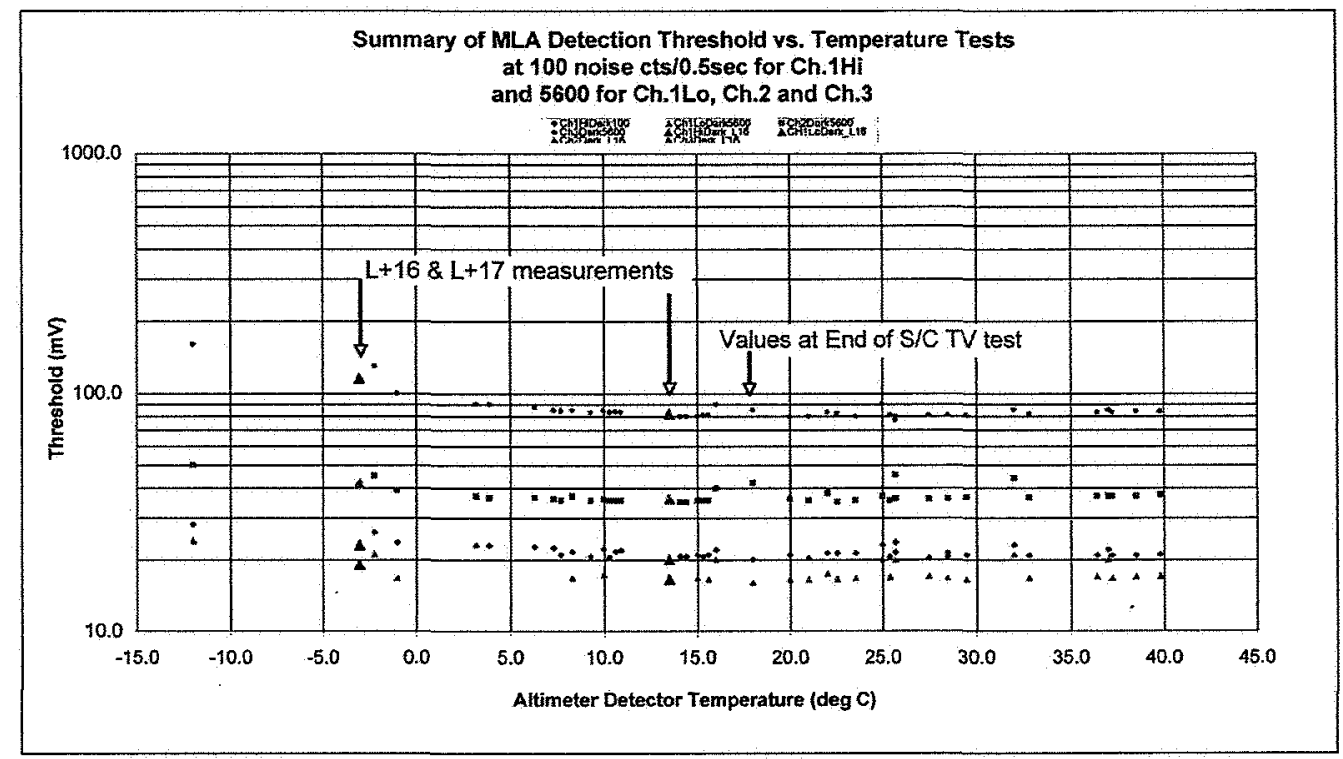

Figure 22 MLA detector noise vs. threshold

On 20 August (Launch +17 days) MLA was commanded to Science mode, causing the laser to fire. Laser performance was normal with the laser energy monitor reporting 19 to $20 \mathrm{~mJ}$ of energy. Figure 23 shows a plot of post-launch laser energy with an equivalent cold turn-on during vacuum testing. The slightly lower energy $(\sim 0.3 \mathrm{~mJ})$ can be attributed to the lack of beam termination at the end of the beam expander. During testing it was observed that the laser energy monitor was sensitive to back reflection from either the beam stop or the beam dump. Since it was impossible to fire the laser unterminated on the ground this condition could not be verified, but the drop in the monitor reading was expected. The matching upward slope after several minutes of operation and the laser diode current pulse width indicate a healthy laser.

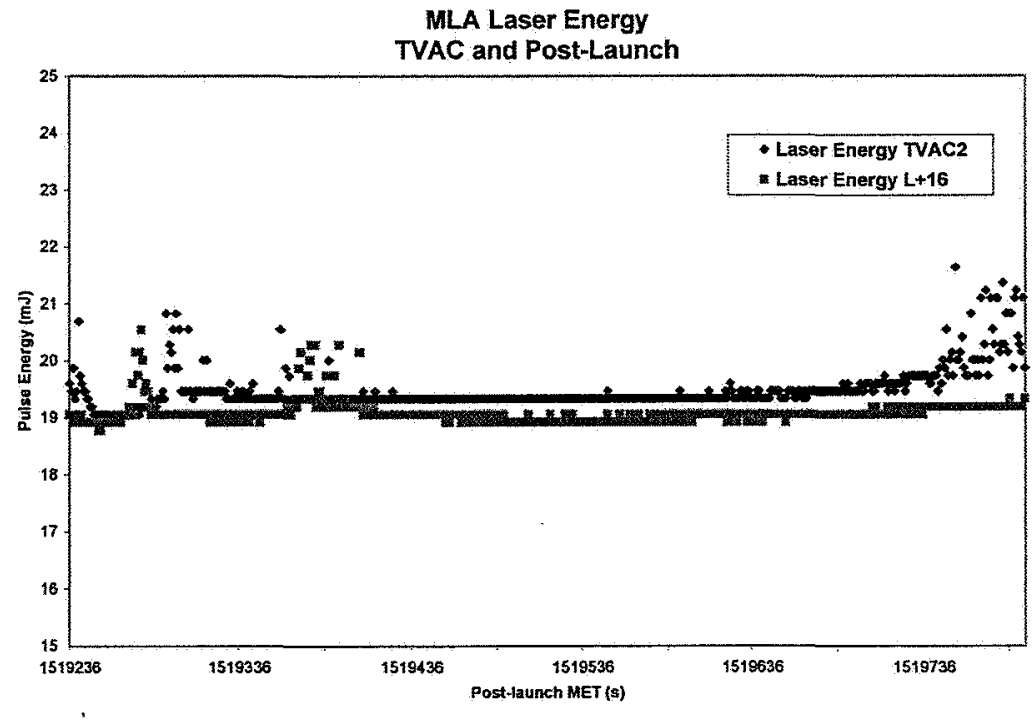

Figure 23 MLA laser energy pre-launch and post-launch 
One unanticipated glitch was the need for the operational laser bench heater during cruise operation of the laser. This heater was switching on a 9-minute period for 4 minutes and added approximately $250 \mathrm{~mA}$ to the total MLA current. Since the spacecraft C\&DH computes power by multiplying the current monitor value times the bus voltage, and the MLA current is only sampled at the $35 \mathrm{~ms}$ peak, the reported power with the heater was $40.8 \mathrm{~W}$. This apparent power draw violated a MESSENGER spacecraft autonomy rule that automatically shuts down any instrument drawing excessive current. This condition was realized just prior to the planned laser turn-on that proceeded with the agreement of the payload systems and spacecraft systems engineers.

\subsection{Cruise Calibration}

In May 2005 MESSENGER successfully performed a sequence of Earth scan maneuvers to calibrate MLA pointing, radiometry and laser pulse timing during cruise. The results of these tests will be published at a later time. A second radiometric calibration will also be performed during the planned Venus fly-by.

\subsection{Data Products}

The time-of-flight range data from MLA will be combined with MESSENGER spacecraft pointing knowledge and Radio Science range and range-rate tracking data obtained from the Deep Space Network to produce Digital Elevation Models of the planet's surface referenced to Mercury's center of mass. These models can then be used to determine and track the planet's topography on a regional and planetary scale to assess libration. Pulse width data from the high and low threshold channels will be used to determine received pulse energy, which along with the transmitted energy measurement will indicate surface reflectivity at $1064 \mathrm{~nm}$.

\subsection{Operation Plans and Observing Strategy}

MLA will operate in Science mode when the spacecraft nadir pointing line-of-sight range to Mercury is less than $1200 \mathrm{~km}$ or the slant range is less than $800 \mathrm{~km}$. These limits results in an operating time of approximately twenty to forty minutes per 12-hour orbit. Depending on the orbit, MLA will be commanded to Standby mode 15 to 480 minutes prior to Science mode to enable the operational heaters on the laser bench and insure that the laser amplifier is at $15^{\circ} \mathrm{C}$ when entering Science mode. During the remainder of each orbit MLA will be commanded to Keep Alive mode to conserve power. There are also eclipse periods during which MLA will be powered off.

\subsection{Summary}

The Mercury Laser Altimeter will provide precise laser pulse time-of-flight measurements that along with MESSENGER spacecraft pointing and Deep Space Network tracking data will be used to determine the detailed topography of Mercury's northern hemisphere, measure topographic profiles across major geologic structures, track large scale topographic features to assess the planet's libration, and measure the surface reflectivity of Mercury at $1064 \mathrm{~nm}$. The successful development, integration, and testing of MLA to date have demonstrated the practicability of its miniaturized laser transmitter, low-cost scalable receiver architecture, and low-power time-of-flight measurement electronics for future space flight missions. 


\section{Acknowledgements}

We wish to thank the following people for their significant contributions to the successful completion of the MLA instrument effort: Ed Amatucci, Adrienne Beamer, Pete Dogoda, Tom Feild, Ron Follas, Ame Fox, Jeff Guzek, Randy Hedgeland, Sid Johnson, Igor Kleyner, Steve Li, Steve Lindauer, Billy Mamakos, Dave McComas, Roger Miller, Tony Miller, Lou Nagao, Karen Pham, Steve Schmidt, Stan Scott, Nancy Stafford, Jon Vermillion, Ken Waggoner, Tony Yu, Ron Zellar, and the MESSENGER team at APL, in particular Steve Jaskulek, Eliot Rodberg, Chuck Schlemm, Stan Kozuch, Jack Ercol and Ted Hartka. 


\section{References}

Degnan, J. J.: 2002, Journal of Geodynamics, 34, 503

Abshire, J.B., Sun, X., Afzal, R.S.: 2000, Applied Optics, 39, 2440

Garvin J., Bufton J., Blair J., Harding D., Luthcke S., Frawley J., Rowlands D.:1998, Physics and Chemistry of the Earth, 23, 1053

Hawkins, S. E., Boldt, J., Darlington, E. H., Espiritu, R., Gold, R., Gotwols, B., Grey, M., Hash, C., Hayes, J., Jaskulek, S., Kardian, C., Keller, M., Malaret, E., Murchie, S. L., Murphy, P., Peacock, K., Prockter, L., Reiter, A., Robinson, M. S., Schaefer, E., Shelton, R., Sterner, R., Taylor, H., Watters, T., Williams, B.: 2005, Space Science Reviews, this issue

Krebs, D. J., Novo-Gradac, A. M., Li, S. X., Lindauer, S. J., Afzal, R. S., Yu, A. W.: 2005, Applied Optics, 44, 1715

Paschalidis, N., Stamatopoulos, N., Karadamoglou, K., Kottaras, G., Paschalidis, V., Sarris, E., McNutt, R., Mitchell, D., McEntire, R.: 2002, IEEE Transactions on Nuclear Science, 49. 1156

Ramos-Izquierdo, L., Scott III, V. S., Schmidt, S., Britt, J., Mamakos, W., Trunzo, R., Cavanaugh, J., Miller, R.: 2005, Applied Optics, 44, 1748

Smith, D. E., Zuber, M. T., Frey, H. V., Garvin, J. B., Head, J. W., Muhleman, D. O., Pettengill, G. H., Phillips, R. J., Solomon, S. C., Zwally, H. J., Banerdt, W. B., Duxbury, T. C.: 1998, Science 279, 1686

Solomon, S. C. McNutt, R. L., Gold, R.E., Acuna, M.H., Baker, D. N., Boyntom, W.V., Chapman, C. R., Cheng, A. F., Gloeckler, G., Head III, J. W., Krimigis, S. M., McLintock, W. E., Murchie, S. L., Peale, S. J., Phillips, R. J., Robinson, M. S., Slavin, J. A., Smith, D. E., Strom, R. G., Trombka, J. L., Zuber, M. T.: 2001, Planetary and Space Science, 49, 1445

Sun, X., Cavanaugh, J. F., Smith, J. C., Bartels, A. E.: 2004, in A. A. Sawchuk (ed.), Trends in Optics and Photonics Series, Conference on Lasers and Electro-Optics (CLEO), 96B, Optical Society of America, Washington, D. C.

Zuber, M. T., Smith, D.E., Solomon, S. C., Muhleman, D. O., Head, J. W., Garvin, J. B., Abshire, J. B., Bufton, J. L.: 1992, J. Geophys. Res., 97, 7781

Zwally, H. J., Schutz, B., Abdalati, W., Abshire, J., Bentley, C., Brenner, A., Bufton, J., Dezio, J., Hancock, D., Harding, D., Herring, T., Minster, B., Quinn, K., Palm, S., Spinhirne, J., Thomas, R.: 2002, Journal of Geodynamics, 34, 405 


\section{Figures and Tables}

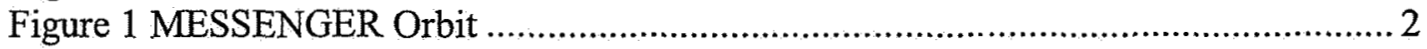

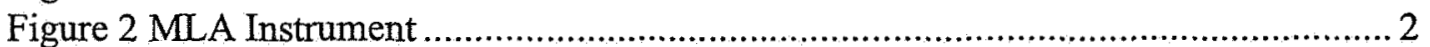

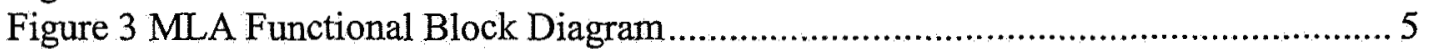

Figure 4 MLA Laser ...................................................................................... 7

Figure 5 MLA Laser Optical Layout ............................................................... 9

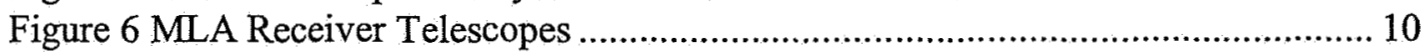

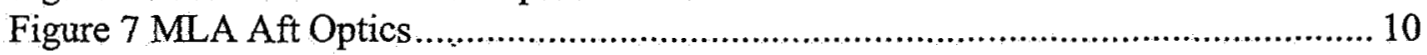

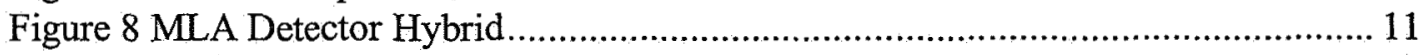

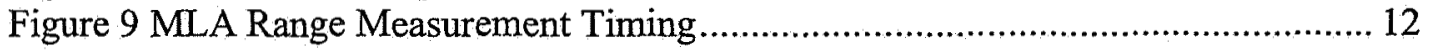

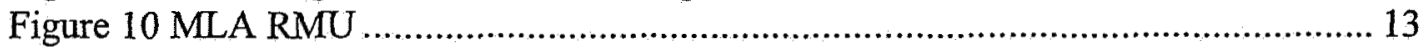

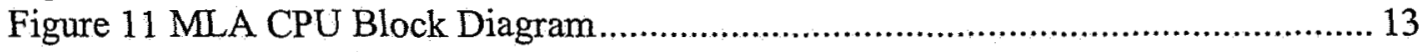

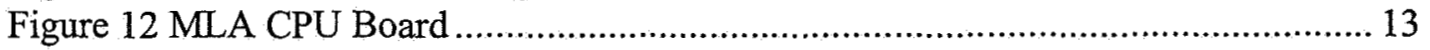

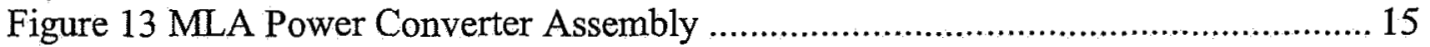

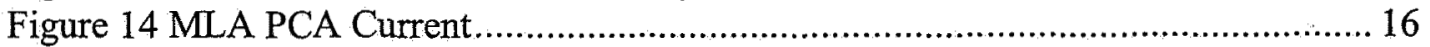

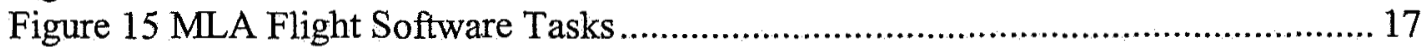

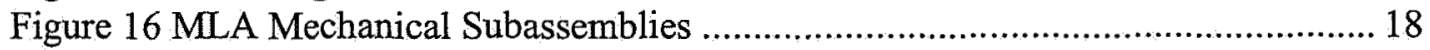

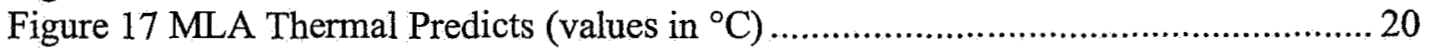

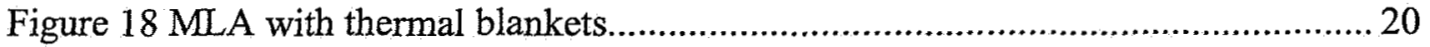

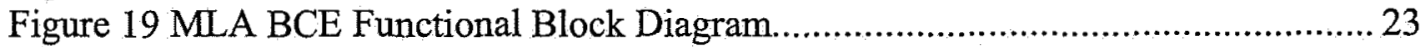

Figure 20 MLA Laser Beam Dump and Alignment Fixture ......................................... 24

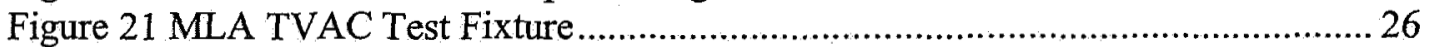

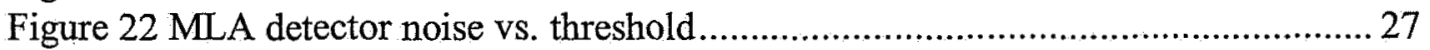

Figure 23 MLA laser energy pre-launch and post-launch....................................... 27

Table 1 MLA Functional Requirements ............................................................... 3

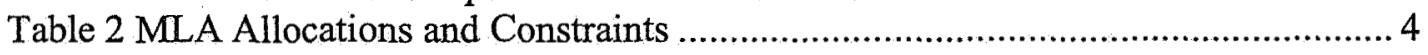

Table 3 MLA Laser Transmitter Requirements .................................................... 8

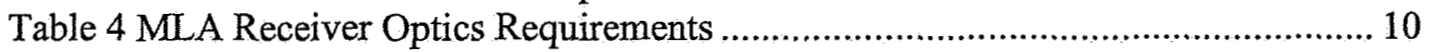

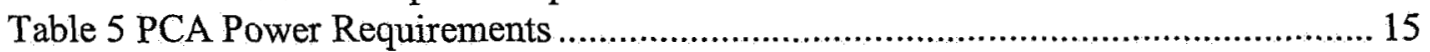

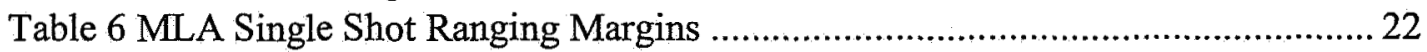

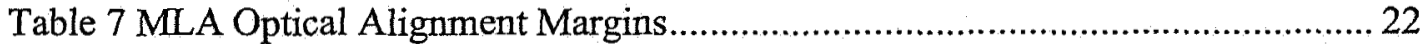

